\title{
Alexander Doktor \\ Global solution of mixed problem for a certain system of nonlinear conservation laws
}

Czechoslovak Mathematical Journal, Vol. 27 (1977), No. 1, 69-95

Persistent URL: http://dml.cz/dmlcz/101447

\section{Terms of use:}

(C) Institute of Mathematics AS CR, 1977

Institute of Mathematics of the Czech Academy of Sciences provides access to digitized documents strictly for personal use. Each copy of any part of this document must contain these Terms of use.

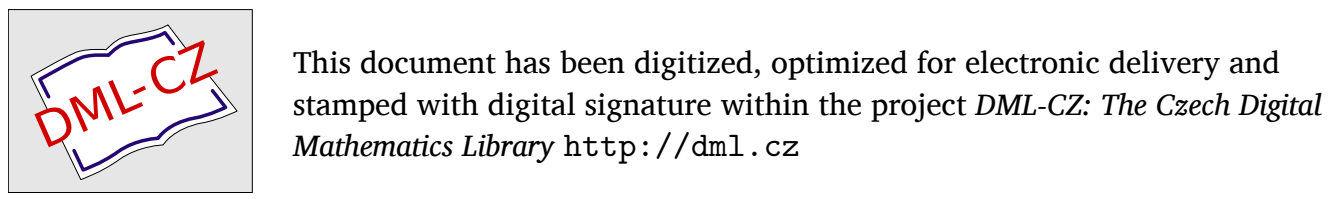




\title{
GLOBAL SOLUTION OF MIXED PROBLEM FOR A CERTAIN SYSTEM OF NONLINEAR CONSERVATION LAWS*)
}

\author{
Alexander Doktor, Praha \\ (Received February 5, 1975)
}

\section{INTRODUCTION}

The present paper deals with the existence of solution of a certain $2 \times 2$ nolinear system of conservation laws on the quadrant $x>0, t>0$, satisfying initial conditions for $t=0, x>0$ and a boundary condition for $x=0, t>0$. As an example of this problem can be taken equations describing the flow of a gas caused by the action of a piston. It is shown that such a mixed problem has a global generalized solution provided the given initial and boundary functions have small variation (see Theorem 2.4). This solution is obtained by means of a difference scheme with a random choice of mesh points. The scheme is similar to that used first by J. GLImM [1] in the case of the Cauchy problem for systems of conservation laws. As well as in this case the proof of convergence of the scheme is based on a detailed investigation of certain functionals representing the norm and variation of approximate solutions (Section 7). However, there are important modifications since we have to take into account the influence of waves reflected on the boundary (Remark 7.3).

\section{FORMULATION OF THE PROBLEM}

We shall consider the following $2 \times 2$ system of conservation laws:

$$
\begin{gathered}
\frac{\partial u}{\partial t}+\frac{\partial}{\partial x} f(u, v)=0, \\
\frac{\partial v}{\partial t}+\frac{\partial}{\partial x} g(u)=0 .
\end{gathered}
$$

*) This paper contains the results of the author's CSc-thesis prepared during his stay at Faculty of Mathematics and Physics, Charles University, Prague. 
Let $F \equiv(f, g) \in C^{(3)}\left(\Omega_{F}\right)$ for a domain $\Omega_{F} \subset \mathrm{R}^{2}$; vectors from $\Omega_{F}$ will be denoted by $U=(u, v), U_{0}=\left(u_{0}, v_{0}\right), \tilde{U}=(\tilde{u}, \tilde{v}), \ldots$ We suppose that the system $(2.1)$ is strictly hyperbolic in the sense that

$$
f_{v}(U)<0, \quad g_{u}(u)<0 \text { for all } U \in \Omega_{F}
$$

(subscripts denote derivatives). Then for each $U \in \Omega_{F}$ the Jacobi matrix $\mathrm{d} F(U)$ of $F$ has two real distinct eigenvalues $\lambda_{1}(U)<\lambda_{2}(U)$. We shall suppose

$$
\lambda_{1}(U)<0<\lambda_{2}(U) ;
$$

(this is the case e.g. for $f=f(v)$ ). The corresponding right eigenvectors of $\mathrm{d} F$ can be written in the form $r^{(1)}=\left(1, a_{1}\right), r^{(2)}=\left(-1, a_{2}\right)$, where $a_{i} \equiv g_{u} \mid \lambda_{i}$. We shall suppose that $(2.1)$ is genuinely nonlinear in the sense of P. D. LAx [2], i.e.

$$
r^{(i)}(U) \operatorname{grad} \lambda_{i}(U)>0, \quad U \in \Omega_{F}, \quad i=1,2 .
$$

Let us denote

$$
\mathrm{R}_{T}^{2} \equiv\left\{(x, t) \in \mathrm{R}^{2} ; t>T\right\} \quad \text { for } \quad T \in \mathrm{R} ; \quad \Omega \equiv\left\{(x, t) \in \mathrm{R}_{0}^{2} ; x>0\right\} .
$$

We shall consider the following problem $(\mathscr{P})$ : Given functions $U_{0}(x), u_{1}(t)$, find a function $U(x, t)$ satisfying the equations $(2.1)$ on $\Omega$, the initial conditions

$$
U(x, 0)=U_{0}(x), \quad x>0,
$$

and the boundary condition

$$
u(0, t)=u_{1}(t), \quad t>0 .
$$

Remark 2.1. The role of $u$ and $v$ is symmetric in the sense that instead of (2.6) we can introduce an analogous boundary condition

$$
v(0, t)=u_{1}(t), \quad t>0 .
$$

The possibility of introducing one boundary condition follows from general considerations about correctness of boundary conditions for hyperbolic systems by which the number of boundary conditions should be equal to the number of characteristics starting on the boundary. Since we suppose (2.3), this number is one.

The form of the boundary condition is then closely related to the Riemann invariants - the functions $w=w(u, v), z=z(u, v)$ with the property $r^{(1)} \operatorname{grad} w=$ $=r^{(2)} \operatorname{grad} z=0$ (see [2], [4]). The classical solution of $(2.1)$ is equivalent to the solution of the system

$$
z_{t}+\lambda_{1} z_{x}=0, \quad w_{t}+\lambda_{2} w_{x}=0 .
$$

Since $z$ is constant along the first characteristics by (2.7) and since $\lambda_{1}<0$, the values $z(0, t)$ are determined by the given initial values $z(x, 0)$. On the other hand $w$ is constant along the second characteristics and since $\lambda_{2}>0$, we can prescribe the 
values of $w$ for $x=0$. From these facts we can deduce the symmetry of $u$ and $v$ : for a given $u(0, t)$ or $v(0, t)$ it suffices to find respectively $v(0, t)$ or $u(0, t))$ such that $z(u(0, t), v(0, t))=z(0, t)$; then we can determine the correct boundary condition for $w: w(0, t)=w(u(0, t), v(0, t))$.

Remark 2.2. If $F$ has the special form

$$
F(u, v)=\left(k v^{-\gamma},-u\right), \quad k>0, \quad \gamma>1,
$$

then (2.1) is the system of gas dynamics describing the one-dimensional isentropic flow of an ideal polytropic gas in Lagrange's coordinates (see e.g. [4]; $u$ means the velocity and $v$ the specific volume) and $F$ satisfies $(2.2)-(2.4)$ on $\Omega_{F}=\{(u, v) ; v>0\}$. In this case the mixed problem $(\mathscr{P})$ has the following physical interpretation: the piston moves by a given speed $u_{1}$ into the gas with the initial state $u_{0}, v_{0}$ and we seek for the resulting flow of the gas. The condition (2.6) would mean that we know the specific volume on the piston, i.e. the pressure on the piston, because $p=k v^{-\gamma}$.

Since, as well as in the case of the Cauchy problem, the nonlinear character of equations (2.1) gives rise to discontinuities of the solution even for smooth $U_{0}, u_{1}$, we shall turn to the generalized solution:

Definition 2.3. Let $\left.U_{0} \in\left[L_{1, l o c}\langle 0, \infty)\right]^{2}, u_{1} \in L_{1, l o c}<0, \infty\right)$ and let

$$
\begin{aligned}
& C_{0}^{2} \equiv\left\{\varphi \in C^{(2)}\left(\mathrm{R}^{2}\right) ; \sup \varphi \text { is compact }\right\}, \\
& C_{00}^{2} \equiv\left\{\psi \in C_{0}^{2} ; \operatorname{supp} \psi \subset\left\{(x, t) \in \mathrm{R}^{2} ; x>0\right\}\right\} .
\end{aligned}
$$

A generalized solution of the problem $(\mathscr{P})$ is any function $U \in\left[L_{1, \text { loc }}(\Omega)\right]^{2}$ such that $F(U) \in\left[L_{1, \text { loc }}(\Omega)\right]^{2}$ and

$$
\begin{gathered}
\int_{\Omega}\left[\psi_{t} u+\psi_{x} f(U)\right] \mathrm{d} x \mathrm{~d} t+\int_{0}^{\infty} \psi(x, 0) u_{0}(x) \mathrm{d} x=0, \quad \psi \in C_{00}^{2}, \\
\int_{\Omega}\left[\varphi_{t} v+\varphi_{x} g(u)\right] \mathrm{d} x \mathrm{~d} t+\int_{0}^{\infty} \varphi(x, 0) v_{0}(x) \mathrm{d} x+ \\
+\int_{0}^{\infty} \varphi(0, t) g\left(u_{1}(t)\right) \mathrm{d} t=0, \quad \varphi \in C_{0}^{2} .
\end{gathered}
$$

It is evident that the classical $C^{(1)}$-solution of $(\mathscr{P})$ is also a generalized solution. On the other hand if $U$ is a generalized solution of $(\mathscr{P})$ such that $U \in C^{(1)}$, a standard argument yields that (2.1), (2.5) hold and, further, $g\left(u_{1}(t)\right)=g(u(0, t)), t>0$. But then (2.6) immediately follows from (2.2) and so $U$ is a classical solution, too.

In what follows we shall use the following notation:

for a vector $V=\left(V_{1}, \ldots, V_{N}\right) \in \mathrm{R}^{N}$, let $|V| \equiv \sum_{i=1}^{N}\left|V_{i}\right|$ be its norm;

for a function $h$, let $\|h\|$ be its $L_{\infty}$-norm. 
The aim of this paper is to prove the following global existence theorem:

Theorem 2.4. Let $F=(f, g)$ satisfy (2.2)-(2.4). Then for each $\tilde{U} \in \Omega_{F}$ there exist constants $d>0, c>0$ such that if $U_{0} \in\left[L_{\infty}\langle 0, \infty)\right]^{2}, u_{1} \in L_{\infty}\langle 0, \infty)$ satisfy

$$
\max \left[\left\|U_{0}-\tilde{U}\right\|,\left\|u_{1}-\tilde{u}\right\|, \underset{\langle 0, \infty)}{\operatorname{Var}} U_{0}, \underset{\langle 0, \infty)}{\operatorname{Var} u_{1}}\right] \leqq d,
$$

then there exists a generalized solution $U \in\left[L_{\infty}(\Omega)\right]^{2}$ of the problem $(\mathscr{P})$. This solution satisfies the inequalities

$$
\begin{aligned}
& \|U-\tilde{U}\| \leqq c\left(\left\|U_{0}-\tilde{U}\right\|+\left\|u_{1}-\tilde{u}\right\|+\underset{\langle 0, \infty)}{\operatorname{Var} U_{0}}+\underset{\langle 0, \infty)}{\operatorname{Var} u_{1}}\right)
\end{aligned}
$$

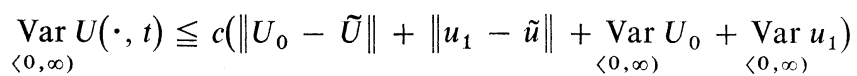

$$
\begin{aligned}
& \text { for each } t \geqq 0 \text {; } \\
& \int_{0}^{\infty}\left|U\left(x, t_{1}\right)-U\left(x, t_{2}\right)\right| \mathrm{d} x \leqq \\
& \leqq c\left|t_{1}-t_{2}\right|\left(\left\|U_{0}-\tilde{U}\right\|+\left\|u_{1}-\tilde{u}\right\|+\underset{\langle 0, \infty)}{\operatorname{Var} U_{0}}+\underset{\langle 0, \infty)}{\operatorname{Var} u_{1}}\right) \\
& \text { for each } t_{1} \geqq 0, \quad t_{2} \geqq 0 \text {. }
\end{aligned}
$$

\section{AUXILIARY RESULTS}

Our construction of approximate solutions is based on simple and shock waves which are particular solutions of the so called initial Riemann problem:

$$
\begin{gathered}
U_{t}+F(U)_{x}=0 \text { for } x \in \mathrm{R}, \quad t>0, \\
U(x, 0)=U_{L} \text { for } x<0, \quad U(x, 0)=U_{R} \text { for } x>0,
\end{gathered}
$$

where $U_{L}, U_{R}$ are given constant vectors from $\Omega_{F}$. We shall refer to this problem briefly as to $\operatorname{IRP}\left(U_{L}, U_{R}\right)$.

The $i$-th simple rarefaction wave (centered at the origin), $i=1,2$, is such a continuous solution of $\operatorname{IRP}\left(U_{L}, U_{R}\right)$ that the $i$-th Riemann invariant is constant in $\mathrm{R}_{0}^{2}$. The $i$-th shock wave, $i=1,2$, is any piece-wise constant solution of $\operatorname{IRP}\left(U_{L}, U_{R}\right)$ with a discontinuity at a half-line $x=\sigma t, t>0$, satisfying the jump conditions

$$
\lambda_{i}\left(U_{R}\right)<\sigma<\lambda_{i}\left(U_{L}\right), \quad \lambda_{i-1}\left(U_{L}\right)<\sigma<\lambda_{i+1}\left(U_{R}\right) .
$$

(The solutions are of course taken in the generalized sense.) Both rarefaction and shock wave shall be called briefly waves. The waves are functions of $x / t$ (the so called automodel functions); the $i$-th rarefaction waves are constant outside the sector 
$\left\{(x, t) \in \mathrm{R}_{0}^{2} ; \lambda_{i}\left(U_{L}\right)<x / t<\lambda_{i}\left(U_{R}\right)\right\}$ (for a more detailed study of the waves see [2], $[3],[4])$. The following "wave parametrization" theorem is known (see [3]):

Theorem 3.1. For each $U_{0} \in \Omega_{F}$ there exist curves $V_{i}\left(U_{0}, u\right), \bar{V}_{i}\left(U_{0}, u\right), i=1,2$, defined and once continuously differentiable for $u$ near to $u_{0}, V_{i}\left(U_{0}, u_{0}\right)=$ $=\bar{V}_{i}\left(U_{0}, u_{0}\right)=v_{0}$, such that $U_{0}$ and $\left(u, V_{i}\left(U_{0}, u\right)\right)\left(U_{0}\right.$ and $\left.\left(u, \bar{V}_{i}\left(U_{0}, u\right)\right)\right)$ can be connected from the left to the right (from the right to the left, respectively) by an $i$-th wave; if we define a parameter $\alpha$ by the relation

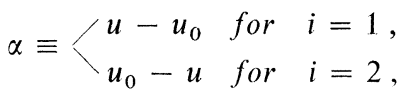

then for $\alpha \geqq 0$ (respectively, $\alpha \leqq 0$ ) this wave is a rarefaction one, otherwise it is a shock.

By means of the curves $V_{i}$ and the parameter $\alpha$ from (3.1) we shall characterize all waves connecting some vectors from the left to the right; so the $i$-th wave $\alpha$ is a rarefaction cne iff $\alpha \geqq 0$ and a shock iff $\alpha<0$.

Since $\lambda_{1}<\lambda_{2}$, the first wave $\alpha_{1}$ connecting $U_{L}$ and $U_{M}$ and the second wave $\alpha_{2}$ connecting $U_{M}$ abd $U_{R}$ can be fitted together to form a solution of $\operatorname{IRP}\left(U_{L}, U_{R}\right)$. We shall say that such a solution is represented by the wave $\alpha \equiv\left(\alpha_{1}, \alpha_{2}\right)$ and write $\alpha: U_{L} \mapsto U_{R}$; the first (second) wave $\alpha$ can be taken as the "vector-wave" $\alpha=(\alpha, 0)$ $(\alpha=(0, \alpha))$.

Theorem 3.2. For each $U_{0} \in \Omega_{F}$ there exist constants $d_{3}\left(U_{0}\right)>0, d_{4}\left(U_{0}\right)>0$ such that for $U_{i} \in \Omega_{F},\left|U_{i}-U_{0}\right| \leqq d_{3}\left(U_{0}\right), i=1,2$, there exists a unique solution of $\operatorname{IRP}\left(U_{1}, U_{2}\right)$ represented by the wave $\varepsilon$ with $|\varepsilon| \leqq d_{4}\left(U_{0}\right)$.

For the proof see [2], [4].

Definition 3.3. Let

$$
\alpha^{+}=\max (0, \alpha), \quad \alpha^{-}=\min (0, \alpha) .
$$

For a sequence (finite or not) of waves $\varepsilon^{1}, \varepsilon^{2}, \ldots$ we define

$$
D\left(\varepsilon^{1}, \varepsilon^{2}, \ldots\right) \equiv \sum_{i<j}\left|\varepsilon_{2}^{i}\right|\left|\varepsilon_{1}^{j}\right|+\sum_{k=1}^{2} \sum_{i \neq j}\left\{\left|\left(\varepsilon_{k}^{i}\right)^{-}\right|\left|\varepsilon_{k}^{j}\right|+\left|\left(\varepsilon_{k}^{i}\right)^{+}\right|\left|\left(\varepsilon_{k}^{j}\right)^{-}\right|\right\} .
$$

Remark 3.4. In the terminology of approaching waves (see [1]) we can write $D\left(\varepsilon^{1}, \ldots\right)$ as follows:

$$
D\left(\varepsilon^{1} \cdot \varepsilon^{2}, \ldots\right)=\sum\left\{\left|\varepsilon_{k}^{i}\right|\left|\varepsilon_{m}^{j}\right| ; \varepsilon_{k}^{i} \text { and } \varepsilon_{m}^{j} \text { approach }\right\} ;
$$

so $D$ "measures" the influence of possible interactions between the waves $\varepsilon^{i}$. (Two waves $\alpha, \beta$ approach (each other) iff they can interact in a finite time.) 
In [1] J. GLimm has proved an important theorem concerning "composing" of Riemann problems. We give here its slight generalization suitable for our purposes:

Theorem 3.5. Let $U_{0} \in \Omega_{F}$ be fixed and let $U_{i} \in \Omega_{F},\left|U_{i}-U_{0}\right| \leqq d_{3}\left(U_{0}\right), i=$ $=1,2,3,4 ; \varepsilon: U_{1} \mapsto U_{4}, \alpha^{i}: U_{i-1} \mapsto U_{i}, i=2,3,4$. Then

$$
\varepsilon_{i}=\alpha_{i}^{2}+\alpha_{i}^{3}+\alpha_{i}^{4}+D\left(\alpha^{2}, \alpha^{3}, \alpha^{4}\right) O(1), \quad i=1,2,
$$

where the function $O(1)$ is uniform in the sense that there exists a constant $K_{1}\left(U_{0}\right)>$ $>0$ such that

$$
|O(1)| \leqq K_{1}\left(U_{0}\right) \text { for all } \alpha^{2}, \alpha^{3}, \alpha^{4} \text { considered } .
$$

"Wave" solutions $U$ of $\operatorname{IRP}\left(U_{L}, U_{R}\right)$ are in general defined a.e. in $\overline{\mathrm{R}}_{0}^{2}$; if we put $U(x, 0) \equiv U_{L}$ for $x \leqq 0, U(x, 0) \equiv U_{R}$ for $x>0$ and $U(x, t) \equiv U(x, t-0)=$ $=\lim _{s \rightarrow t-0} U(x, s)$ on a possible line of discontinuity, $U$ is defined everywhere in $\overline{\mathbf{R}}_{0}^{2}$.

For two different points $a, b \in \mathrm{R}^{2}$ we denote by $\{a-b\}$ the segment with the endpoints $a, b$.

Definition 3.6. Let $\left\{a^{1}-a^{2}\right\} \subset \overline{\mathrm{R}}_{0}^{2}, a^{i}=\left(a_{x}^{i}, a_{t}^{i}\right), i=1,2$, and let the wave $\alpha$ represent the solution $U$ of $\operatorname{IRP}\left(U_{L}, U_{R}\right)$ for some $U_{L}, U_{R}$. Then the wave $\alpha \cap$ $\cap\left\{a^{1}-a^{2}\right\}$ is defined as follows:

If $a_{x}^{1}, \mid a_{t}^{1}<a_{x}^{2} / a_{t}^{2}$ and if $\alpha$ coincides with an $i$-th wave $\alpha, i \in\{1,2\}$, then

for $\alpha<0$, set $\alpha \cap\left\{a^{1}-a^{2}\right\} \equiv 0$ if $U\left(a^{1}\right)=U\left(a^{2}\right)$ and $\alpha \cap\left\{a^{1}-a^{2}\right\} \equiv \alpha$ otherwise;

for $\alpha \geqq 0$ there exists a rarefaction wave $\beta: U\left(a^{1}\right) \mapsto U\left(a^{2}\right), 0 \leqq \beta \leqq \alpha$; set $\alpha \cap\left\{a^{1}-a^{2}\right\} \equiv \beta$.

Further

$$
\begin{aligned}
& \alpha \cap\left\{a^{1}-a^{2}\right\} \equiv\left\langle\begin{array}{ll}
\alpha \cap\left\{a^{2}-a^{1}\right\} & \text { for } a_{x}^{1} / a_{t}^{1}>a_{x}^{2} / a_{t}^{2}, \\
0 & \text { for } a_{x}^{1} / a_{t}^{1}=a_{x}^{2} / a_{t}^{2}
\end{array} ;\right. \\
& \alpha \cap\left\{a^{1}-a^{2}\right\} \equiv\left(\alpha_{1} \cap\left\{a^{1}-a^{2}\right\},\right. \\
& \left.\alpha_{2} \cap\left\{a^{1}-a^{2}\right\}\right) .
\end{aligned}
$$

For $a, b \in\{t=0\}$ set $\alpha \cap\{a-b\} \equiv \alpha \cap\{\tilde{a}-\tilde{b}\}$, where $\tilde{a}, \tilde{b}$ are such points that $U(\tilde{a})=U(a), U(\tilde{b})=U(b)$.

For $\ell=\left\{a^{0}-a^{1}\right\} \cup\left\{a^{1}-a^{2}\right\}, a^{i} \in \overline{\mathrm{R}}_{0}^{2}, \quad i=0,1,2$, such that $a_{x}^{i-1} / a_{t}^{i-1} \leqq$ $\leqq a_{x}^{i} / a_{t}^{i}, i=1,2$, set $\alpha \cap \ell \equiv \alpha \cap\left\{a^{0}-a^{2}\right\}$.

The total variation $\operatorname{Var} \varphi$ of a function $\varphi$ over the interval $\langle x, y\rangle \subset \mathrm{R}$ is defined as usual, moreover,

$$
\underset{\langle x, \infty)}{\operatorname{Var}} \varphi \equiv \sup _{y>x} \operatorname{Var} \varphi,
$$


For $\varphi$ defined on $\mathrm{R}^{2},\{a-b\} \subset \mathrm{R}^{2}$, let the variation of $\varphi$ along the segment $\{a-b\}$ be

where

$$
\underset{\{a-b\}}{\operatorname{Var} \varphi} \equiv \underset{\langle 0,1\rangle}{\operatorname{Var} \varphi^{*}}
$$

$$
\varphi^{*}(\xi) \equiv \varphi\left(a_{x}+\xi\left(b_{x}-a_{x}\right), a_{t}+\xi\left(b_{t}-a_{t}\right)\right), \quad \xi \in\langle 0,1\rangle .
$$

Lemma 3.7. Let $U_{0} \in \Omega_{F}$. Then there exist constants $c_{i}\left(U_{0}\right)>0, i=1,2$, $d_{8}\left(U_{0}\right) \in\left(0, d_{3}\left(U_{0}\right)>\right.$ with the following properties: for all $U_{i} \in \Omega_{F}$ such that $\left|U_{i}-U_{0}\right| \leqq d_{3}\left(U_{0}\right), i=1,2$, and for the solution $U$ of $\operatorname{IRP}\left(U_{1}, U_{2}\right)$ given by the wave $\varepsilon: U_{1} \mapsto U_{2}$ it holds

$$
\begin{gathered}
\left|U(x, t)-U_{i}\right| \leqq|\varepsilon| / c_{1}\left(U_{0}\right), \quad(x, t) \in \mathrm{R}_{0}^{2}, \quad i=1,2 ; \\
c_{1}\left(U_{0}\right)\left|U_{1}-U_{2}\right| \leqq|\varepsilon| \leqq c_{2}\left(U_{0}\right)\left|U_{1}-U_{2}\right| .
\end{gathered}
$$

If moreover $\left|U_{i}-U_{0}\right| \leqq d_{8}\left(U_{0}\right), i=1,2$, then

$$
\left|U(x, t)-U_{0}\right| \leqq d_{3}\left(U_{0}\right) \text { for all }(x, t) \in \mathrm{R}_{0}^{2}
$$

and for each segment $\{a-b\} \subset \overline{\mathrm{R}}_{0}^{2}$ we have

$$
c_{1}\left(U_{0}\right) \underset{\{a-b\}}{\operatorname{Var}} U \leqq|\varepsilon \cap\{a-b\}| \leqq c_{2}\left(U_{0}\right) \underset{\{a-b\}}{\operatorname{Var} U}
$$

We omit the proof since it is not difficult and follows from the definitions and the automodel character of $U$.

\section{MIXED RIEMANN PROBLEM}

We can pose a problem analogous to the initial-value Riemann problem: to find a solution $U$ of the system (2.1) on $\Omega$ satisfying the initial condition $U(x, 0)=U_{0}$, $x>0$, and the boundary condition $u(0, t)=u_{1}, t>0$, where $U_{0}, u_{1}$ are given constants. In what follows we shall refer to such a problem as to a mixed Riemann problem or briefly $\operatorname{MRP}\left(u_{1}, U_{0}\right)$.

Theorem 4.1. Let $\tilde{U} \in \Omega_{F}$. Then there exists a constant $c^{*}(\tilde{U})>0$ such that if $U_{0} \in \Omega_{F}, u_{1} \in \mathrm{R}$ satisfy $\left|U_{0}-\tilde{U}\right| \leqq d_{3}(\tilde{U}),\left|u_{1}-\tilde{u}\right| \leqq d_{3}(\tilde{U})$ then there exists a solution $U$ of $M R P\left(u_{1}, U_{0}\right)$ given by the second wave $\varepsilon$ and satisfying the inequality

$$
\left|v(0, t)-v_{0}\right| \leqq c^{*}(\tilde{U})|\varepsilon|, \quad t>0 .
$$

Proof. Let $U_{1} \equiv\left(u_{1}, \bar{V}_{2}\left(U_{0}, u_{1}\right)\right)$. There exists a solution $U^{*}$ of $\operatorname{IRP}\left(U_{1}, U_{0}\right)$ given by the second wave $\varepsilon=u_{0}-u_{1}$; for $U^{*}$ we have

$$
u^{*}(0, t)=u_{1}, \quad t>0 ; \quad U^{*}(x, 0)=U_{0}, \quad x>0 .
$$


Hence it is sufficient to put $\left.U \equiv U^{*}\right|_{\Omega}$. (Geometrically the solution of $\operatorname{MRP}\left(u_{1}, U_{0}\right)$ is represented by the intersection $U_{1}$ of the curve $\bar{V}_{2}\left(U_{0}, \cdot\right)$ with the line $u=u_{1}$.)

Since $v(0, t)=v_{1}$ for all $t>0$, we have

$$
\left|v(0, t)-v_{0}\right|=\left|\bar{V}_{2}\left(U_{0}, u_{1}\right)-\bar{V}_{2}\left(U_{0}, u_{0}\right)\right|
$$

and consequently it suffices to put

$$
c^{*}(\tilde{U}) \equiv \sup \left\{\left|\frac{\partial \bar{V}_{2}}{\partial u}(U, \bar{u})\right| ;|U-\tilde{U}| \leqq d_{3}(\tilde{U}),|\bar{u}-\tilde{u}| \leqq d_{3}(\tilde{U})\right\} .
$$

In what follows we shall consider only solutions of $M R P$ of the type constructed in the proof of Theorem 4.1. This agreement makes our considerations unambiguous although no uniqueness is established.

Remark 4.2. Since all results from Sections 3,4 are invariant with respect to translation of the origin, we can apply them to the problem

$$
\begin{gathered}
U_{t}+F(U)_{x}=0 \text { on } \mathrm{R}_{T}^{2}, \quad T \geqq 0 ; \\
U(x, T)=U_{L} \text { for } x<X, \quad U(x, T)=U_{R} \text { for } x>X, \quad X \in \mathrm{R} .
\end{gathered}
$$

We shall call such a problem $\operatorname{IRP}\left(U_{L}, U_{R}\right)$ with the center $(X, T)$; analogously for $M R P$ and waves arising from these problems.

\section{DIFFERENCE SCHEME AND APPROXIMATE SOLUTIONS}

In what follows we shall confine ourselves to certain local character of initial and boundary functions in the sense that for certain fixed $\tilde{U} \in \Omega_{F}$ and sufficiently small constant $d_{50}>0$ we suppose

$$
\max \left[\left\|U_{0}-\tilde{U}\right\|,\left\|u_{1}-\tilde{u}\right\|, \underset{\langle 0, \infty)}{\operatorname{Var}} U_{0}, \underset{\langle 0, \infty)}{\operatorname{Var} u_{1}}\right] \leqq d_{50} .
$$

Let us start from the restriction

$$
d_{50} \leqq d_{8}(\tilde{U})
$$

and denote $d_{3} \equiv d_{3}(\tilde{U}), d_{8} \equiv d_{8}(\tilde{U}), c^{*} \equiv c^{*}(\tilde{U})$. Further let

$$
\begin{aligned}
& \lambda_{0}^{*} \equiv \sup \left\{\left|\lambda_{2}(U)\right| ;|U-\tilde{U}| \leqq\left(1+c^{*}\right) d_{3}\right\}, \\
& \lambda_{0} \equiv \sup \left\{\left|\lambda_{j}(\Phi(U, \boldsymbol{\alpha}))\right| ;|U-\tilde{U}| \leqq d_{3},|\boldsymbol{\alpha}| \leqq d_{4}, j=1,2\right\},
\end{aligned}
$$

where

$$
\Phi(U, \alpha) \equiv\left(u+\alpha_{1}-\alpha_{2}, V_{2}\left(\left(u+\alpha_{1}, V_{1}\left(U, u+\alpha_{1}\right)\right), u+\alpha_{1}-\alpha_{2}\right)\right) .
$$


We fix a constant $q_{0}>0$ such that

$$
q_{0}>2 \max \left[\lambda_{0}, \lambda_{0}^{*}\right]
$$

and from now on we shall suppose that $r>0, s>0$ satisfy

$$
\stackrel{r}{s}=q_{0}
$$

so that $s=s(r)$ is a function of $r$.

Let $\mathrm{N}=\{1,2, \ldots\}$ be the set of all natural numbers, $\bar{N} \equiv\{0\} \cup \mathrm{N}$.

Now we define

$$
Y \equiv\left\{(m, n) \in \overline{\mathrm{N}}^{2} ; m+n \text { is even }\right\}
$$

and for $(m, n) \in Y$ let $A_{m, n}=A_{m, n}(r) \subset \bar{\Omega}$ be the horizontal line-segment

$$
A_{m, n} \equiv\{(x, n s) ; \max [0,(m-1) r] \leqq x \leqq(m+1) r\} .
$$

Finally, let

$$
A=A(r) \equiv \prod_{(m, n) \in Y} A_{m, n}(r)
$$

An infinite vector $a=\left\{a_{m, n}\right\} \in A$ will represent a random choice of mesh points in our difference scheme.

Given $r>0$ and $a \in A(r)$, the approximate solution $U_{r, a}$ of the mixed problem $(\mathscr{P})$ is constructed as follows:

$1^{\circ}$ Let

$$
\begin{array}{ll}
U_{0, r}(x) \equiv U_{0}(m r) & \text { for } \quad x \in(\max [0,(m-1) r],(m+1) r\rangle, \quad m \in \overline{\mathrm{N}} \text { even } \\
u_{1, r}(t) \equiv u_{1}(n s) & \text { for } \quad t \in\langle(n-1) s,(n+1) s), \quad n \in \mathrm{N} \quad \text { odd }
\end{array}
$$

and further

$$
U_{r, a}(x, 0) \equiv U_{0, r}(x) \text { for } x>0 \text {. }
$$

Then (5.1) implies

$$
\left|U_{r, a}\left(a_{m, n}\right)-\tilde{U}\right| \leqq d_{3}
$$

for $n=0, m \in \overline{\mathrm{N}}$ even.

$2^{\circ}$ Suppose that for $n \in \overline{\mathrm{N}}$ the approximate solution $U_{r, a}$ is defined for $t \leqq n s$, $x \geqq 0$ and that (5.7) holds for all $m \in \bar{N}, m+n$ even. Let us denote

$$
m_{0}=m_{0}(n) \equiv \min \{m \in \overline{\mathrm{N}} ;(m, n) \in Y\} .
$$

By (5.7) and Theorem 3.2, for each $m \geqq m_{0}+2,(m, n) \in Y$ there exists a solution $U^{m, n}$ of $\operatorname{IRP}\left(U_{r, a}\left(a_{m-2, n}\right), U_{r, a}\left(a_{m, n}\right)\right)$ with the center $((m-1) r, n s)$ given by the wave

$$
\varepsilon^{m, n}: U_{r, a}\left(a_{m-2, n}\right) \mapsto U_{r, a}\left(a_{m, n}\right)
$$


with the same center. We define

$$
\begin{gathered}
U_{r, a}(x, t) \equiv U^{m, n}(x, t) \text { for } t \in\langle n s,(n+1) s), \\
x \in\left(\max \left[\frac{r}{2},(m-2) r\right], m r\right\rangle, m \geqq m_{0}+2 .
\end{gathered}
$$

Further, by Theorem 4.1 there exists a solution $U^{m_{0}, n}$ of $\operatorname{MRP}\left(u_{1, r}(n s), U_{r, a}\left(a_{m_{0}, n}\right)\right)$ with the center $(0, n s)$ given by the second wave $\varepsilon^{m_{0}, n}$. Then we define

$$
\begin{gathered}
U_{r, a}(x, t) \equiv U^{m_{0}, n}(x, t) \quad \text { for } x \in\left\langle 0, \quad \max \left[\frac{r}{2}, m_{0} r\right]\right\rangle, \\
t \in\langle n s,(n+1) s) .
\end{gathered}
$$

Relations (5.9), (5.10) define $U_{r, a}$ on the strip

$$
\Pi_{n} \equiv\{(x, t) \in \bar{\Omega} ; t \in\langle n s,(n+1) s)\} .
$$

$3^{\circ}$ At the mesh points on the line $t=(n+1) s$ we define

$$
U_{r, a}\left(a_{m, n+1}\right) \equiv U_{r, a}\left(a_{m, n+1}^{\prime},(n+1) s-0\right), \quad(m, n+1) \in Y,
$$

where $a_{m, n}^{\prime}$ denotes the $x$-coordinate of the point $a_{m, n}$.

$4^{\circ}$ On the line $t=(n+1) s$ we set

$$
U_{r, a}(x,(n+1) s) \equiv U_{r, a}\left(a_{m, n+1}\right) \text { for } x \in(\max [0,(m-1) r],(m+1) r>.
$$

$5^{\circ}$ Relations (5.9) $-(5.13)$ define $U_{r, a}$ on $\bar{\Pi}_{n}$ and we can repeat our construction from the item $2^{\circ}$ provided (5.7) holds for $n+1$ and all $m$ such that $m+n+1$ is even.

If (5.7) holds for all $(m, n) \in Y$ then the approximate solution $U_{r, a}$ is defined on the whole quadrant $\bar{\Omega}$; this will be proved for sufficiently small $d_{50}$ in the following sections. The initial restriction (5.2) implies that $U_{r, a}$ is defined at least for $t \leqq 2 s$.

Lemma 5.1. The waves $\varepsilon^{m, n},(m, n) \in Y$ do not intersect each other in the strip $\bar{\Pi}_{n}$ and consequently, $U_{r, a}$ is an exact solution of the system (2.1) in the strip $\Pi_{n}$.

Proof of Lemma follows immediately from conditions (5.3), (5.4) and from the basic properties of rarefaction and shock waves.

\section{6. $\mathscr{I}$-CURVES}

By an $\mathscr{I}$-curve we mean, roughly speaking, an infinite piece-wise linear curve starting on the line $x=0$ and connecting the mesh points $a_{m, n}, a_{m+1, \bar{n}}, \bar{n}=n \pm 1$. 
Definition 6.1. Let $b_{n} \equiv\left(0,\left(n+\frac{1}{2}\right) s\right), n \in \bar{N}$. For a given $n \in \bar{N}$ let the first segment of an $\mathscr{I}$-curve $\mathscr{I}$ starting at its origin $b_{n}$ be one of the following segments: $\left\{b_{n}-a_{m_{0}(n), n}\right\},\left\{b_{n}-a_{m_{0}(n+1), n+1}\right\}\left(m_{0}(n)\right.$ is defined by (5.8)). If we have defined a part $\mathscr{I}^{*}$ of $\mathscr{I}$ and

$$
\begin{gathered}
m=\max \left\{\bar{m} ; a_{\bar{m}, n} \in \mathscr{I}^{*}\right\}, \text { then the next segment of } \mathscr{I} \text { is } \\
\left\{a_{m, n}-a_{m+1, n+1}\right\} \text { or }\left\{a_{m, n}-a_{m+1, n-1}\right\} .
\end{gathered}
$$

The mesh points $a_{m, n} \in \mathscr{I}, b_{n} \in \mathscr{I}$ are called vertices of $\mathscr{I}$, the set of all vertices is denoted by $V(\mathscr{I})$.

We shall confine our consideration to $\mathscr{I}$-curves lying in the strip $\bar{\Pi}_{n} \cup \bar{\Pi}_{n+1}$ for some $n \in \overline{\mathrm{N}}$ and denote by $[\mathscr{I}]$ the set of all such curves.

Finally, the first $\mathscr{I}$-curve $\mathcal{O} \in[\mathscr{I}]$ is defined as

$$
\mathcal{O} \equiv\left\{b_{0}-a_{0,0}\right\} \cup\left\{a_{0,0}-a_{1,1}\right\} \cup\left\{a_{1,1}-a_{2,0}\right\} \cup \ldots, \quad \mathcal{O} \subset \bar{\Pi}_{0} .
$$

Each $\mathscr{I}$-curve $\mathscr{I} \in[\mathscr{I}]$ divides the domain $\left\{(x, t) \in \mathrm{R}^{2} ; x>0\right\}$ into two components; we denote $\Omega(\mathscr{I})$ the one of them for which $\Omega(\mathscr{I}) \supset\{(x, t) ; x>0, t<0\}$.

Definition 6.2. Let $\mathscr{I}, \mathscr{J} \in[\mathscr{I}]$ be two $\mathscr{I}$-curves. We say that $\mathscr{I} \leqq \mathscr{J}$ iff $\Omega(\mathscr{I}) \subset$ $\subset \Omega(\mathscr{J})$ (i.e. $\mathscr{J}$ lies toward the larger time then $\mathscr{I}$ ).

Lemma 6.3. $1^{\circ}$ The set $[\mathscr{I}]$ is partially ordered by the relation $\leqq$.

$2^{\circ}$ The curve $\mathcal{O}$ is the least element of $[\mathscr{I}]$, i.e. $\mathcal{O}<\mathscr{J}$ for all $\mathscr{J} \in[\mathscr{I}], \mathscr{J} \neq \mathcal{O}$.

$3^{\circ}$ The set $[\mathscr{I}]$ satisfies the condition of minimality, i.e. each nonempty subset $B \subset[\mathscr{I}]$ has a minimal element - such a curve $\mathscr{I}_{B} \in B$ that for $\mathscr{I} \in B$ it is not $\mathscr{I}<\mathscr{I}_{B}$.

Proof of the first two items of Lemma follows immediately from the definitions. As to the item $3^{\circ}$, one of minimal elements of $B$ can be constructed by induction as follows: Let

$$
\begin{gathered}
n_{0} \equiv \min \left\{n \in \overline{\mathrm{N}} ; b_{n} \in \mathscr{I} \in B\right\}, \quad n_{1} \equiv \min \left\{n \in \overline{\mathrm{N}} ;\left\{b_{n_{0}}-a_{m, n}\right\} \subset \mathscr{I} \in B\right\} ; \\
\ell_{1} \equiv\left\{b_{n_{0}}-a_{m_{0}\left(n_{1}\right), n_{1}}\right\} .
\end{gathered}
$$

Evidently $\ell_{1} \subset \mathscr{I}$ for some $\mathscr{I} \in B$. Having constructed segments $\ell_{i}=\left\{a_{m_{i}-1, n_{i}-1}-\right.$ $\left.\left.-a_{m_{i}, n_{i}}\right\}^{*}\right), i=1,2, \ldots, k$, we put

$$
\begin{aligned}
& n_{k+1} \equiv \min \left\{n \in \overline{\mathrm{N}} ; a_{m_{k}+1, n} \in \mathscr{I} \in B, \bigcup_{i=1}^{k} \ell_{i} \subset \mathscr{I}\right\}, \\
& \ell_{k+1} \equiv\left\{a_{m_{k}, n_{k}}-a_{m_{k}+1, n_{k+1}}\right\} .
\end{aligned}
$$

*) Here formally $a_{m_{1-1, n_{0}}}=b_{n_{0}}$. 
Evidently, the $\mathscr{I}$-curve $\mathscr{I}_{B} \equiv \bigcup_{i=1}^{\infty} \ell_{i}$ belongs to $B$. If $\mathscr{I}_{B}$ were not a minimal element of $B$, then there would exist $\mathscr{I}^{*} \in B$ and $k^{*} \in \mathrm{N}$ such that $\mathscr{I}^{*}<\mathscr{I}_{B}$ and $\bigcup_{i=1}^{k^{*}} \ell_{i} \subset \mathscr{I}^{*}$, $\ell_{k^{*}+1} \notin \mathscr{I}^{*}$. But this would contradict to the definition of $n_{k^{*}+1}$.

Definition 6.4. Let $\mathscr{I}, \mathscr{J} \in[\mathscr{I}]$ be two $\mathscr{I}$-curves. We say that $\mathscr{J}$ is the successor of $\mathscr{I}$ iff one of the following possibilities holds:

$$
\begin{array}{rlrl}
V(\mathscr{J}) \backslash V(\mathscr{I})=a_{m, n}, & & V(\mathscr{I}) \backslash V(\mathscr{I})=a_{m, n-2}, \quad(m, n) \in Y ; \\
V(\mathscr{J}) \backslash V(\mathscr{I})=\emptyset, & & V(\mathscr{I}) \backslash V(\mathscr{J})=a_{0, n} \text { or } \\
V(\mathscr{J}) \backslash V(\mathscr{I})=a_{0, n}, & & V(\mathscr{I}) \backslash V(\mathscr{J})=\emptyset, & n \text { even } ; \\
V(\mathscr{J}) \backslash V(\mathscr{I})=b_{n+1}, & & V(\mathscr{I}) \backslash V(\mathscr{J})=b_{n}, \quad n \in \overline{\mathrm{N}} .
\end{array}
$$

\section{FUNCTIONALS $F_{1}, F_{2}$}

We shall introduce real functions $F_{i}:[\mathscr{I}] \rightarrow\langle 0, \infty\rangle, i=1,2$, which, in a sense, represent apirori estimates of the variation and the norm of the approximate solution. Since in this section we shall deal only with fixed $r, a$ we shall write briefly $U(x, t)$ instead of $U_{r, a}(x, t)$.

Definition 7.1. Let the approximate solution $U$ be defined along the curve $\mathscr{I} \in[\mathscr{I}]$. We define the waves $\varepsilon^{m, n} \cap \mathscr{I},(m, n) \in Y$ as follows:

$1^{\circ}$ For $m \geqq 1$ let $\left.\ell^{*} \equiv \mathscr{I} \cap\left[\left\{a_{m-2, n}-a_{m-1, n+1}\right\} \cup\left\{a_{m-1, n+1}-a_{m, n}\right\}\right]^{*}\right)$. If $\ell^{*} \neq \emptyset$, we put $\varepsilon^{m, n} \cap \mathscr{I} \equiv \varepsilon^{m, n} \cap \ell^{*}$ (see Definition 3.6).

$2^{\circ}$ For $m=1$ and $\ell^{*}=\emptyset$ let $\ell_{1} \equiv \mathscr{I} \cap\left\{b_{n}-a_{1, n}\right\}$. If $\ell_{1} \neq \emptyset$, we put $\varepsilon^{1, n} \cap \mathscr{I} \equiv$ $\equiv \varepsilon^{1, n} \cap l_{1}=\varepsilon^{1, n}$.

$3^{\circ}$ For $m=0$ and $n$ even the curve $\mathscr{I}$ can "come back" into the wave $\varepsilon^{0, n}$ and can intersect this wave even three times. Therefore we define $\varepsilon^{0, n} \cap \mathscr{I}$ as a set of one or two waves taking into account only the segments of $\mathscr{I}$ intersecting $\boldsymbol{\varepsilon}^{0, \boldsymbol{n}}$ in the direction of increasing $x$ :

$3 \mathrm{a}^{\circ}$ If $\left\{b_{n}, a_{0, n}, a_{1, n+1}, a_{2, n}\right\} \subset V(\mathscr{I})$ and $a_{1, n+1}^{\prime}<r / 2$ then

$$
\varepsilon^{0, n} \cap \mathscr{I} \equiv\left\{\varepsilon^{0, n}, \varepsilon^{0, n} \cap\left\{a_{1, n+1}-a_{2, n}\right\}\right\} .
$$

$3 \mathrm{~b}^{\circ}$ If $\left\{b_{n}, a_{1, n+1}, a_{2, n+2}\right\} \subset V(\mathscr{I})$ and $a_{0, n} \notin \mathscr{I}$ then

$$
\varepsilon^{0, n} \cap \mathscr{I} \equiv\left\{\varepsilon^{0, n} \cap\left\{b_{n}-a_{1, n+1}\right\}\right\} .
$$

*) Here formally $a_{-1, n}=b_{n}$. 
$3 c^{\circ}$ If $\left\{b_{n+1}, a_{1, n+1}, a_{2, n}\right\} \subset V(\mathscr{I})$ or $\left\{b_{n-1}, a_{0, n}, a_{1, n+1}, a_{2, n}\right\} \subset V(\mathscr{I})$ and if $a_{1, n+1}^{\prime}<r / 2$ then

$$
\varepsilon^{0, n} \cap \mathscr{I} \equiv\left\{\varepsilon^{0, n} \cap\left\{a_{1, n+1}-a_{2, n}\right\}\right\} .
$$

$3 \mathrm{~d}^{\circ}$ If $\left\{b_{n}, a_{0, n}, a_{1, n-1}\right\} \subset V(\mathscr{I}), a_{1, n+1}^{\prime} \geqq r / 2$ or if $\left\{b_{n}, a_{0, n}, a_{1, n-1}\right\} \subset V(\mathscr{I})$ or if $\left\{b_{n}, a_{1, n+1}, a_{2, n}\right\} \subset V(\mathscr{I}), a_{0, n} \notin \mathscr{I}$ or if $\left\{b_{n}, a_{0, n}, a_{1, n+1}, a_{2, n+2}\right\} \subset V(\mathscr{I})$ then

$$
\varepsilon^{0, n} \cap \mathscr{I} \equiv\left\{\varepsilon^{0, n}\right\} \text {. }
$$

$4^{\circ}$ In all other cases except $1^{\circ}-3^{\circ}$ we take $\varepsilon^{m, n} \cap \mathscr{I}$ as an empty (nonexisting) wave (e.g. if $\varepsilon^{m, n}$ is not defined or if $\mathscr{I} \cap \Pi_{n}=\emptyset$ ).

For $\mathscr{I} \in[\mathscr{I}]$ we denote by $\varepsilon(\mathscr{I})$ the set of all nonempty waves $\varepsilon^{m, n} \cap \mathscr{I}$ intersecting the curve $\mathscr{I}$. We arrange the countable set $\varepsilon(\mathscr{I})$ into a sequence $\left\{\alpha^{i}\right\}_{i=1}^{\infty}=\varepsilon(\mathscr{I})$ in such a way that $i>j$ iff $\alpha^{i}$ intersects $\mathscr{I}$ after (toward the larger $x$ than) $\alpha^{j}$.

Definition 7.2. Let $U$ be defined along $\mathscr{I} \in[\mathscr{I}], \varepsilon(\mathscr{I})=\left\{\alpha^{i}\right\}_{i=1}^{\infty}, \alpha^{i}=\left(\alpha_{1}^{i}, \alpha_{2}^{i}\right)$. We define the following functionals:

$$
\begin{aligned}
& L(\mathscr{I}) \equiv \sum_{i=1}^{\infty}\left|\alpha^{i}\right| ; \\
& Q(\mathscr{I}) \equiv D\left(\alpha^{1}, \alpha^{2}, \ldots\right)+\sum_{i<j}\left\{\left|\left(\alpha_{1}^{i}\right)^{+}\right|\left|\alpha^{j}\right|+\left|\left(\alpha_{1}^{i}\right)^{-}\right|\left(\left|\alpha_{1}^{j}\right|+\left|\left(\alpha_{2}^{j}\right)^{+}\right|\right)\right\} ; \\
& F_{1}(\mathscr{I}) \equiv L(\mathscr{I})+14 K_{1} Q(\mathscr{I}) ;
\end{aligned}
$$

where $D$ has been introduced in Definition 3.3 and $K_{1}=K_{1}(\tilde{U})>0$ is the constant from Theorem 3.5 .

Remark 7.3. As follows from the proof of Lemma 7.4, the functional $L$ represents the variation of the approximate solution $U$. However, suitable estimates cannot be proved directly for $L$, which is why certain "correction" $14 K_{1} Q$ is added to $L$.

The functional $Q$ can be interpreted as the influence of the interactions of waves from the approximate solution: $D$ represents the waves which can interact directly (see Remark 3.4) while the other terms on the right-hand side of (7.2) represent the influence of waves reflecting on the boundary $x=0$ (i.e. on the head of the piston in the physical interpretation of Remark 2.2). Since $\lambda_{1}<0$, the waves of the first family intersect the line $x=0$ at a finite time $T>0$; for $t>T$ the solution of the mixed problem has the character of a wave of the second family of the opposite kind - i.e., a rarefaction wave if the initial wave is a shock and a shock wave if the initial wave is a rarefaction one (see e.g. [4] for the equations of gas dynamics). To the interaction of reflected waves, we can then apply again Remark 3.4.

Lemma 7.4. Let $U$ be defined along $\mathscr{I} \in[\mathscr{I}]$. Then

$$
F_{1}(\mathscr{I}) \leqq\left[1+28 K_{1} L(\mathscr{I})\right] L(\mathscr{I}) .
$$


If, moreover, $\mathscr{I} \subset \bar{\Pi}_{n}$ for some $n \in \overline{\mathrm{N}}$ and

$$
|U(b)-\tilde{U}| \leqq d_{8} \text { for all } b \in V(\mathscr{I}),
$$

then

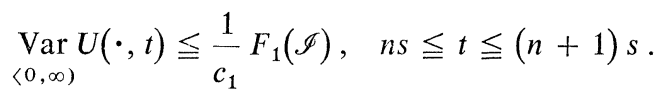

Finally,

$$
F_{1}(\mathcal{O}) \leqq c_{11}\left[\underset{\langle 0, \infty)}{\operatorname{Var}} U_{0}+\left\|U_{0}-\tilde{U}\right\|+\left\|u_{1}-\tilde{u}\right\|\right],
$$

where

$$
c_{1.1} \equiv\left(2+c_{2}\right)\left(1+84 K_{1}\left(2+c_{2}\right) d_{50}\right) .
$$

$\left(c_{i} \equiv c_{i}(\tilde{U}), i=1,2\right.$, are the constants from Lemma 3.7.)

Proof. Evidently $D\left(\alpha^{1}, \alpha^{2}, \ldots\right) \leqq \sum_{i, j=1}^{\infty}\left|\alpha^{i}\right|\left|\alpha^{j}\right|=[L(\mathscr{I})]^{2}$ and analogously for the other terms in (7.2). Therefore $Q(\mathscr{I}) \leqq 2[L(\mathscr{I})]^{2}$ and we immediately obtain (7.4). Further, if $\mathscr{I} \subset \bar{\Pi}_{n}, n \in \overline{\mathrm{N}}$, then

$$
L(\mathscr{I}) \geqq \sum_{m}\left|\varepsilon^{m, n}\right| .
$$

Using Lemma 3.7 we get for $t \in\langle n s,(n+1) s)$

$$
\left|\varepsilon^{m, n}\right| \geqq c_{1} \underset{\langle\max [0,(m-2) r], m r\rangle}{\operatorname{Var}} U(\cdot, t) .
$$

Since $L(\mathscr{I}) \leqq F(\mathscr{I}),(7.9)$ and (7.10) imply (7.6). Finally

$$
L(\mathcal{O}) \leqq 2\left|\varepsilon^{0,0}\right|+\sum_{k=1}^{\infty}\left|\varepsilon^{2 k, 0}\right|
$$

and since $\left|\varepsilon^{0,0}\right|=\left|u_{0}(0)-u_{1}(s)\right|, U(\cdot, 0)=U_{0, r}$, we obtain by means of Lemma 3.7

$$
L(\mathcal{O}) \leqq\left(2+c_{2}\right)\left[\underset{\langle 0, \infty)}{\operatorname{Var}} U_{0, r}+\left|u_{0}(0)-u_{1}(s)\right|\right]
$$

and (7.7) follows by direct computation. (Let us observe that the inequality in (7.9) can hold iff $n$ is even and $\varepsilon^{0, n} \cap \mathscr{I} \neq\left\{\varepsilon^{0, n}\right\}$, so in all other cases we have moreover $L(\mathscr{I}) \leqq c_{2} \operatorname{Var} U(\cdot, t)$.)

Definition 7.5. Let $U$ be defined along $\mathscr{I} \in[\mathscr{I}]$. For $a_{m_{0}, n_{0}} \in \mathscr{I}$ let $\varepsilon\left(a_{m_{0}, n_{0}} ; \mathscr{I}\right)$ be the set of all waves $\alpha \cap \ell$ where $\alpha \in \varepsilon(\mathscr{I})$ and $\ell \subset \mathscr{I}$ is a segment of the form 
$\left\{a_{m-1, n^{*}}-a_{m, n}\right\}$ or $\left\{b_{n^{*}}-a_{m, n}\right\}, m \leqq m_{0}$. For $b_{n} \in \mathscr{I}$ let $\varepsilon\left(b_{n} ; \mathscr{I}\right) \equiv \emptyset$. Further let $b \in V(\mathscr{I})$. We put

$$
\begin{gathered}
L(b ; \mathscr{I}) \equiv \sum_{\alpha \in \varepsilon(b ; I)}|\alpha|, \\
F_{2}(b ; \mathscr{I}) \equiv c_{9}|U(b)-\tilde{U}|+L(b ; \mathscr{I})+14 K_{1} Q(\mathscr{I}),
\end{gathered}
$$

where

$$
c_{9} \equiv \min \left[c_{1},\left(1+c^{*}\right)^{-1}\right]
$$

Finally let

$$
F_{2}(\mathscr{I}) \equiv \sup _{b \in V(I)} F_{2}(b ; \mathscr{I})
$$

Lemma 7.6. Let $U$ be defined along $\mathscr{I} \in[\mathscr{I}]$. Then

$$
|U(b)-\tilde{U}| \leqq \frac{1}{c_{9}} F_{2}(\mathscr{I})
$$

for each $b \in V(\mathscr{I})$. Moreover, if (7.5) holds then (7.15) is true for all $b \in \mathscr{I}$. Finally

$$
F_{2}(\mathcal{O}) \leqq c_{12}\left(\left\|U_{0}-\tilde{U}\right\|+\left\|u_{1}-\tilde{u}\right\|+\underset{\langle 0, \infty)}{\operatorname{Var} U_{0}}\right),
$$

where

$$
c_{12} \equiv c_{9}+2 c_{11} .
$$

Proof. Inequality (7.15) for $b \in V(\mathscr{F})$ follows directly from the definition of $F_{2}$. Let $(x, t) \in \mathscr{I}$ and suppose $(x, t) \in \ell$, where $\ell=\left\{a_{m-1, n^{\prime}}-a_{m, n}\right\}$ or $\ell=$ $=\left\{b_{n^{\prime}}-a_{m, n}\right\}\left|n-n^{\prime}\right| \leqq 1$. Let e.g. (for the sake of definiteness) $n^{\prime}=n+1$ and put $\alpha \equiv \varepsilon^{m, n} \cap \ell$. Then

$$
\left|U(x, t)-U\left(a_{m, n}\right)\right| \leqq \underset{t}{\operatorname{Var}} U \leqq \frac{1}{c_{1}}|\alpha|
$$

by Lemma 3.7 and therefore

$$
|U(x, t)-\tilde{U}| \leqq \frac{1}{c_{1}}|\alpha|+\left|U\left(a_{m, n}\right)-\tilde{U}\right|
$$

Evidentely $\left.\alpha \in \varepsilon\left(a_{m, n} ; \mathscr{I}\right) *\right)$ so that (7.18) implies

$$
c_{9}|U(x, t)-\tilde{U}| \leqq L\left(a_{m, n} ; \mathscr{I}\right)+c_{9}\left|U\left(a_{m, n}\right)-\tilde{U}\right| \leqq F_{2}\left(a_{m, n} ; \mathscr{I}\right)
$$

*) For $m=0, \alpha \notin \varepsilon\left(a_{m, n} ; \mathscr{I}\right)$ may occur, but in this case $\varepsilon^{0, n} \in \varepsilon\left(a_{m, n} ; \mathscr{I}\right),\left|\varepsilon^{0, n}\right| \geqq|\boldsymbol{\alpha}|$ and so it suffices to replace $\alpha$ by $\varepsilon^{0, n}$. 
and consequently (7.15) holds for $b=(x, t)$. Finally, since $d_{50} \leqq d_{8}$ we can apply the preceding estimates to the curve $\mathcal{O}$, in particular for $b=a_{m, 1}$ we have $c_{9}\left|U\left(a_{m, 1}\right)-\widetilde{U}\right| \leqq F_{2}\left(a_{m+1,0} ; \mathcal{O}\right)$ and therefore

$$
F_{2}(\mathcal{O}) \leqq c_{9} \sup _{k \in \overline{\mathrm{N}}}\left|U\left(a_{2 k, 0}\right)-\tilde{U}\right|+2 F_{1}(\mathcal{O})
$$

because $L(b ; \mathcal{O}) \leqq L(\mathcal{O})$. Then $(7.16)$ is obtained by means of Lemma 7.4.

Lemma 7.7. Let $\mathscr{J} \in[\mathscr{I}]$ be a successor of $\mathscr{I} \in[\mathscr{I}]$ of the type (6.1). Let $U$ be defined along $\mathscr{I}$ so that (7.5) holds and let further

$$
\begin{gathered}
F_{1}(\mathscr{I}) \leqq c_{3}(\mathscr{I}) d_{50}, \quad c_{3}(\mathscr{I})>0, \\
d_{50} \leqq \min \left[d_{8},\left(c_{3}(\mathscr{I}) 14 K_{1}\right)^{-1}\right] .
\end{gathered}
$$

Then $U$ is defined also along $\mathscr{J}$ and

$$
F_{i}(\mathscr{J}) \leqq F_{i}(\mathscr{I}), \quad i=1,2 .
$$

Proof. The curves $\mathscr{I}, \mathscr{J}$ differ in the diamond $A$ with the vertices $a_{m, n}, a_{m, n-2}$, $a_{m-1, n-1}, a_{m+1, n-1}$. From (7.5) it follows that there exists a solution of $\operatorname{IRP}\left(U\left(a_{m-1, n-1}\right), U\left(a_{m+1, n-1}\right)\right)$ and so $U$ is defined along $\mathscr{J}$.

While the upper part $\mathscr{J} \backslash \mathscr{I}$ of $\Lambda$ is intersected by one wave $\varepsilon^{m+1, n-1}$, the lower part $\mathscr{I} \backslash \mathscr{J}$ of $\Lambda$ can be intersected by two or three waves. However, the proofs are similar in both cases. Let us consider the case $m=2, a_{1, n-1}^{1}<r / 2$, i.e. the case of three waves. Denote $\varepsilon(\mathscr{I})=\left\{\boldsymbol{\alpha}^{i}\right\}, \varepsilon(\mathscr{J})=\left\{\boldsymbol{\beta}^{i}\right\}$ and $\boldsymbol{\gamma} \equiv \boldsymbol{\varepsilon}^{0, n-2} \cap\left\{a_{1, n-1}-a_{2, n-2}\right\}$, $\boldsymbol{\delta} \equiv \varepsilon^{4, n-2} \cap\left\{a_{2, n-2}-a_{3, n-1}\right\}, \boldsymbol{\beta} \equiv \varepsilon^{3, n-1}, \boldsymbol{\varepsilon} \equiv \varepsilon^{2, n-2} ; D \equiv D(\gamma, \boldsymbol{\varepsilon}, \boldsymbol{\delta})$.

Comparing $\varepsilon(\mathscr{J})$ and $\varepsilon(\mathscr{I})$ we see that $\boldsymbol{\beta}=\boldsymbol{\beta}^{2}$ and

$$
\begin{aligned}
L(\mathscr{J})= & L(\mathscr{I})+|\boldsymbol{\beta}|-|\gamma|-|\boldsymbol{\varepsilon}|-|\boldsymbol{\delta}| \\
Q(\mathscr{J})= & Q(\mathscr{I})-D+\left|\beta_{2}^{1}\right|\left(\left|\beta_{1}\right|-\left|\varepsilon_{1}\right|-\left|\delta_{1}\right|\right)+ \\
& +\left|\left(\beta_{2}^{1}\right)^{-}\right|\left(\left|\beta_{2}\right|-\left|\gamma_{2}\right|-\left|\varepsilon_{2}\right|-\left|\delta_{2}\right|\right)+ \\
& +\left|(\beta)_{2}^{1+}\right|\left(\left|\left(\beta_{2}\right)^{-}\right|-\left|\left(\gamma_{2}\right)^{-}\right|-\left|\left(\varepsilon_{2}\right)^{-}\right|-\left|\left(\delta_{2}\right)^{-}\right|\right)+ \\
& +\left(\left|\left(\beta_{1}\right)^{-}\right|-\left|\left(\varepsilon_{1}\right)^{-}\right|-\left|\left(\delta_{1}\right)^{-}\right|\right) \sum_{k=3}^{\infty}\left|\beta_{1}^{k}\right|+ \\
& +\left(\left|\left(\beta_{1}\right)^{+}\right|-\left|\left(\varepsilon_{1}\right)^{+}\right|-\left|\left(\delta_{1}\right)^{+}\right|\right) \sum_{k=3}^{\infty}\left|\left(\beta_{1}^{k}\right)^{-}\right|+ \\
& +\left(\left|\beta_{2}\right|-\left|\gamma_{2}\right|-\left|\varepsilon_{2}\right|-\left|\delta_{2}\right|\right) \sum_{k=3}^{\infty}\left|\beta_{1}^{k}\right|+ \\
& +\left(\left|\left(\beta_{2}\right)^{-}\right|-\left|\left(\gamma_{2}\right)^{-}\right|-\left|\left(\varepsilon_{2}\right)^{-}\right|-\left|\left(\delta_{2}\right)^{-}\right|\right) \sum_{k=3}^{\infty}\left|\beta_{2}^{k}\right|+
\end{aligned}
$$




$$
\begin{aligned}
& +\left(\left|\left(\beta_{2}\right)^{+}\right|-\left|\left(\gamma_{2}\right)^{+}\right|-\left|\left(\varepsilon_{2}\right)^{+}\right|-\left|\left(\delta_{2}\right)^{+}\right|\right) \sum_{k=3}^{\infty}\left|\left(\beta_{2}^{k}\right)^{-}\right|+ \\
& +\left(\left|\left(\beta_{1}\right)^{+}\right|-\left|\left(\varepsilon_{1}\right)^{+}\right|-\left|\left(\delta_{1}\right)^{+}\right|\right) \sum_{k=3}^{\infty}\left|\boldsymbol{\beta}^{k}\right|+ \\
& +\left(\left|\left(\beta_{1}\right)^{-}\right|-\left|\left(\varepsilon_{1}\right)^{-}\right|-\left|\left(\delta_{1}\right)^{-}\right|\right) \sum_{k=3}^{\infty}\left(\left|\beta_{1}^{k}\right|+\mid\left(\beta_{2}^{k}\right)^{-}\right)+ \\
& +\left(-\left|\left(\varepsilon_{1}\right)^{+}\right||\boldsymbol{\delta}|-\left|\left(\varepsilon_{1}\right)^{-}\right|\left[\left|\delta_{1}\right|+\left|\left(\delta_{2}\right)^{-}\right|\right]\right) \equiv \\
& \equiv Q(\mathscr{I})-D+q_{1}+q_{2}+\ldots+q_{11} .
\end{aligned}
$$

By Theorem 3.5 we obtain $\beta_{i}=\gamma_{i}+\varepsilon_{i}+\delta_{i}+D O(1), i=1,2$, and consequently

$$
\Delta_{i} \equiv\left|\beta_{i}\right|-\left|\gamma_{i}\right|-\left|\varepsilon_{i}\right|-\left|\delta_{i}\right| \leqq D K_{1}, \quad i=1,2 .
$$

Further if $\left(\beta_{i}\right)^{-} \neq 0$ then

$$
\begin{aligned}
q_{i}^{\prime} & \equiv\left|\left(\beta_{i}\right)^{-}\right|-\left|\left(\gamma_{i}\right)^{-}\right|-\left|\left(\varepsilon_{i}\right)\right|^{-}|-|\left(\delta_{i}\right)^{-}\left|=-\beta_{i}-\right|\left(\gamma_{i}\right)^{-} \mid-\ldots= \\
& =-\gamma_{i}-\varepsilon_{i}-\delta_{i}-D O(1)-\left|\left(\gamma_{i}\right)^{-}\right|-\ldots \leqq \\
& \leqq\left|\left(\gamma_{i}\right)^{-}\right|+\left|\left(\varepsilon_{i}\right)^{-}\right|+\left|\left(\delta_{i}\right)^{-}\right|+D|O(1)|-\left|\left(\gamma_{i}\right)^{-}\right|-\ldots
\end{aligned}
$$

and therefore

$$
q_{i}^{\prime} \leqq D K_{1}, \quad i=1,2,
$$

since evidently $q_{i}^{\prime} \leqq 0$ for $\left(\beta_{i}\right)^{-}=0$. Analogously (it is $\gamma_{1}=0$ )

$$
\left|\left(\beta_{1}\right)^{+}\right|-\left|\left(\varepsilon_{1}\right)^{+}\right|-\left|\left(\delta_{1}\right)^{+}\right| \leqq D K_{1} .
$$

Inequalities (7.25)-(7.26) imply

$$
q_{j} \leqq D K_{1} L(\mathscr{I}), \quad j=1,2, \ldots, 10,
$$

and since $q_{11} \leqq 0$, we get

$$
Q(\mathscr{J}) \leqq Q(\mathscr{I})+\left(10 K_{1} L(\mathscr{I})-1\right) D .
$$

Then we obtain from (7.25), (7.28)

$$
\Delta_{1}+\Delta_{2}+14 K_{1} Q(\mathscr{J}) \leqq\left[2+140 K_{1} L(\mathscr{I})-14\right] K_{1} D+14 K_{1} Q(\mathscr{I}) .
$$

In virtue of (7.19), (7.20)

$$
2+140 K_{1} L(\mathscr{I})-14 \leqq 2+10-14<0,
$$

and therefore

$$
|\boldsymbol{\beta}|-|\gamma|-|\boldsymbol{\varepsilon}|-|\boldsymbol{\delta}|+14 K_{1} Q(\mathscr{J}) \leqq 14 K_{1} Q(\mathscr{I}) .
$$


Similarly $Q(\mathscr{J}) \leqq Q(\mathscr{I})+D\left[10 K_{1} c_{3}(\mathscr{I}) d_{50}-1\right] \leqq Q(\mathscr{I})+D[(10 / 14)-1]$ and so

$$
Q(\mathscr{J}) \leqq Q(\mathscr{I})
$$

Now (7.21) for $i=1$ immediately follows from (7.22) and (7.31).

Taking account of (7.31), (7.32) one can easily show that

$$
F_{2}(b ; \mathscr{J}) \leqq F_{2}(b ; \mathscr{I}) \text { for } \quad b \in V(\mathscr{I}) \cap V(\mathscr{J})
$$

If we denote $\varphi^{1} \equiv \varepsilon^{3, n-1} \cap\left\{a_{1, n-1}-a_{2, n}\right\}, \varphi^{2} \equiv \varepsilon^{3, n-1} \cap\left\{a_{2, n}-a_{3, n-1}\right\}$, then

$$
\begin{gathered}
L\left(a_{2, n} ; \mathscr{J}\right)=L\left(a_{3, n-1} ; \mathscr{I}\right)+\left|\varphi^{1}\right|-|\gamma|-|\varepsilon|-|\delta|, \\
\left|U\left(a_{2, n}\right)-\tilde{U}\right| \leqq\left|U\left(a_{3, n-1}\right)-\tilde{U}\right|+\left|\varphi^{2}\right| \mid c_{1} .
\end{gathered}
$$

Since $\left|\varphi^{1}\right|+\left|\varphi^{2}\right|=|\boldsymbol{\beta}|$, we obtain by means of (7.31)

$$
\begin{gathered}
F_{2}\left(a_{2, n} ; \mathscr{J}\right) \leqq c_{9}\left|U\left(a_{3, n-1}\right)-\tilde{U}\right|+L\left(a_{3, n-1} ; \mathscr{I}\right)+ \\
+|\boldsymbol{\beta}|-|\gamma|-|\boldsymbol{\varepsilon}|-|\boldsymbol{\delta}|+14 K_{1} Q(\mathscr{J}) \leqq F_{2}\left(a_{3, n-1} ; \mathscr{I}\right) .
\end{gathered}
$$

Now (7.21) for $i=2$ follows from (7.33), (7.34).

Lemma 7.8. Let $\mathscr{J} \in[\mathscr{I}]$ be a successor of $\mathscr{I} \in[\mathscr{I}]$ of the type (6.2) and let $U$ be defined along $\mathscr{I}$. Then $U$ is defined also along $\mathscr{I}$ and (7.21) holds.

Proof. Since $V(\mathscr{J}) \subset V(\mathscr{I})$ or $V(\mathscr{J})=V(\mathscr{J}) \cup\left\{a_{0, n}\right\}, \mathscr{I}=\left\{b_{n-1}-a_{1, n-1}\right\} \cup$ $\cup[\mathscr{J} \cap \mathscr{I}], U$ is defined along $\mathscr{J}$. Comparing $\varepsilon(\mathscr{I}), \varepsilon(\mathscr{J})$ we see that $\varepsilon(\mathscr{I})=$ $=\varepsilon(\mathscr{J}) \cup\left\{\varepsilon^{0, n} \cap\left\{a_{0, n}-a_{1, n+1}\right\}\right\}$ for $V(\mathscr{J}) \backslash V(\mathscr{I})=\emptyset, a_{1, n+1}^{1}<r / 2$, otherwise $\varepsilon(\mathscr{J})=\varepsilon(\mathscr{I})$. Hence the rest of the proof is easy.

Lemma 7.9. Let $\mathscr{J} \in[\mathscr{I}]$ be a successor of $\mathscr{I} \in[\mathscr{I}]$ of the type (6.3) for $n$ even and let $U$ be defined along $\mathscr{I}$ such that

$$
\left|U\left(a_{1, n+1}\right)-\tilde{U}\right| \leqq d_{3} .
$$

Then $U$ is defined also along $\mathscr{J}$ and (7.21) holds.

Proof. We have $\mathscr{J} \backslash \mathscr{I}=\left\{b_{n+1}-a_{1, n+1}\right\}, \mathscr{I} \backslash \mathscr{J}=\left\{b_{n}-a_{1, n+1}\right\}, n$ even. Since by Theorem 4.1 there exists a solution of $\operatorname{MRP}\left(u_{1}((n+1) s), U\left(a_{1, n+1}\right)\right)$, $U$ is defined along $\mathscr{J}$. Denote $\varepsilon(\mathscr{I})=\left\{\alpha^{i}\right\}_{i=1}^{\infty}, \varepsilon(\mathscr{J})=\left\{\boldsymbol{\beta}^{i}\right\}_{i=1}^{\infty}$ and

$$
\gamma \equiv\left\langle\begin{array}{ll}
\alpha^{2}=\varepsilon^{2, n} \cap\left\{b_{n}-a_{1, n+1}\right\} & \text { if } a_{1, n+1}^{\prime}>r / 2, \\
0 & \text { otherwise }
\end{array}\right.
$$


Evidently $\boldsymbol{\alpha}^{1}=\varepsilon^{0, n} \cap\left\{b_{n}-a_{1, n+1}\right\}, \boldsymbol{\beta}^{1}=\varepsilon^{1, n+1}$. Comparing $\varepsilon(\mathscr{I}), \varepsilon(\mathscr{J})$ we see that

$$
\begin{aligned}
L(\mathscr{J})= & L(\mathscr{I})+\left|\boldsymbol{\beta}^{1}\right|-\left|\alpha^{1}\right|-|\gamma| \\
Q(\mathscr{J})= & Q(\mathscr{I})-D\left(\boldsymbol{\alpha}^{1}, \gamma\right)+\left[\left|\left(\beta_{2}^{1}\right)^{-}\right|-\left|\left(\alpha_{2}^{1}\right)^{-}\right|-\left|\left(\gamma_{2}\right)^{-}\right|\right] \sum_{k=2}^{\infty}\left|\boldsymbol{\beta}^{k}\right|+ \\
& +\left[\left|\left(\beta_{2}^{1}\right)^{+}\right|-\left|\left(\alpha_{2}^{1}\right)^{+}\right|-\left|\left(\gamma_{2}\right)^{+}\right|\right] \sum_{k=2}^{\infty}\left[\left|\beta_{1}^{k}\right|+\left|\left(\beta_{2}^{k}\right)^{-}\right|\right]- \\
& -\left|\left(\gamma_{1}\right)^{-}\right| \sum_{k=2}^{\infty}\left[2\left|\beta_{1}^{k}\right|+\left|\left(\beta_{2}^{k}\right)^{-}\right|\right]- \\
& -\left|\left(\gamma_{1}\right)^{+}\right| \sum_{k=2}^{\infty}\left[\left|\left(\beta_{1}^{k}\right)^{-}\right|+\left|\boldsymbol{\beta}^{k}\right|\right]= \\
\equiv & Q(\mathscr{I})-D\left(\alpha^{1}, \gamma\right)+q_{1}+q_{2}+q_{3}+q_{4} .
\end{aligned}
$$

Since $a_{1, n+1}^{\prime} \leqq r / 2$ implies $\boldsymbol{\alpha}^{1}=\boldsymbol{\beta}^{1}$ and $\boldsymbol{\gamma}=0$, we can confine ourselves to the case $a_{1, n+1}^{\prime}>r / 2$. By the definition of $U, u_{1 r}$ we have $u\left(b_{n+1}\right)=u\left(b_{n}\right)=u_{1}((n+1) s)$. Hence

and further

$$
\alpha_{2}^{1}=u\left(b_{n}\right)-u\left(a_{0, n}\right), \quad \beta_{2}^{1}=u\left(b_{n}\right)-u\left(a_{1, n+1}\right)
$$

$$
\gamma_{1}=u(r,(n+1 / 2) s)-u\left(a_{0, n}\right), \quad \gamma_{2}=u(r,(n+1 / 2) s)-u\left(a_{1, n+1}\right) .
$$

Therefore $\beta_{2}^{1}=\alpha_{2}^{1}-\gamma_{1}+\gamma_{2}$ and

$$
\begin{gathered}
\left|\left(\beta_{2}^{1}\right)^{-}\right|=\max \left(0,-\beta_{2}^{1}\right) \leqq\left|\left(\alpha_{2}^{1}\right)^{-}\right|+\left|\left(\gamma_{1}\right)^{+}\right|+\left|\left(\gamma_{2}\right)^{-}\right|, \\
\left|\left(\beta_{2}^{1}\right)^{+}\right|=\max \left(0, \beta_{2}^{1}\right) \leqq\left|\left(\alpha_{2}^{1}\right)^{+}\right|+\left|\left(\gamma_{1}\right)^{-}\right|+\left|\left(\gamma_{2}\right)^{+}\right|, \\
\left|\boldsymbol{\beta}^{1}\right| \leqq\left|\boldsymbol{\alpha}^{1}\right|+|\gamma| .
\end{gathered}
$$

Now (7.38), (7.39) imply $q_{1}+q_{4} \leqq 0, q_{2}+q_{3} \leqq 0$ and hence

$$
Q(\mathscr{J}) \leqq Q(\mathscr{I})
$$

Since $L(\mathscr{J}) \leqq L(\mathscr{I})$ by $(7.36)$ and $(7.40)$, (7.41) yields $F_{1}(\mathscr{J}) \leqq F_{1}(\mathscr{I})$. Further $L(b ; \mathscr{J})=L(b ; \mathscr{I})+\left|\boldsymbol{\beta}^{1}\right|-\left|\boldsymbol{\alpha}^{1}\right|-|\gamma|$ for $b \in V(\mathscr{I}) \cap V(\mathscr{J})$ and therefore using (7.40), (7.41) we see that

$$
F_{2}(b ; \mathscr{J}) \leqq F_{2}(b ; \mathscr{I})
$$

for $b \in V(\mathscr{I}) \cap V(\mathscr{J})$. Since $L\left(b_{n+1} ; \mathscr{J}\right)=L\left(a_{1, n+1} ; \mathscr{I}\right)-\left|\boldsymbol{\alpha}^{1}\right|-|\gamma|$, we obtain from (4.1)

$$
\begin{gathered}
F_{2}\left(b_{n+1} ; \mathscr{J}\right) \leqq c_{9}\left|U\left(a_{1, n+1}\right)-\tilde{U}\right|+c_{9}\left(1+c^{*}\right)\left|\boldsymbol{\beta}^{1}\right|+ \\
\quad+L\left(a_{1, n+1} ; \mathscr{I}\right)-\left|\boldsymbol{\alpha}^{1}\right|-|\gamma|+14 K_{1} Q(\mathscr{J}) .
\end{gathered}
$$

But $c_{9}\left(1+c^{*}\right) \leqq 1$ and so (7.40), (7.41), (7.43) imply (7.42) for $b=b_{n+1}$. Hence $F_{2}(\mathscr{J}) \leqq F_{2}(\mathscr{I})$ and the proof is complete. 
Lemma 7.10. Let $\mathscr{J} \in[\mathscr{I}]$ be a successor of $\mathscr{I} \in[\mathscr{I}]$ of the type (6.3) for $n$ odd and let $U$ be defined along $\mathscr{I}$ such that

$$
\left|U\left(a_{0, n+1}\right)-\tilde{U}\right| \leqq d_{3}
$$

and (7.19), (7.20) hold. Then $U$ is defined also along $\mathscr{J}$ and

$$
F_{i}(\mathscr{J}) \leqq F_{i}(\mathscr{I})+2\left|u_{1}((n+2) s)-u_{1}(n s)\right|, \quad i=1,2 .
$$

Proof. Since $\mathscr{J} \backslash \mathscr{I}=\left\{b_{n+1}-a_{0, n+1}\right\}, \mathscr{I} \backslash \mathscr{J}=\left\{b_{n}-a_{0, n+1}\right\}, n$ odd and since (7.44) holds, Theorem 4.1 implies that $U$ is defined at $b_{n+1}$ and hence along $\mathscr{J}$. Let $\gamma \equiv \boldsymbol{\varepsilon}^{1, n} \cap\left\{b_{n}-a_{0, n}\right\}, \varepsilon(\mathscr{I})=\left\{\alpha^{i}\right\}, \varepsilon(\mathscr{J})=\left\{\boldsymbol{\beta}^{i}\right\}$;evidently $\varepsilon^{0, n+1}=\boldsymbol{\beta}^{1}$. Then

$$
\beta_{2}^{1}=\gamma_{2}+u\left(b_{n+1}\right)-u\left(b_{n}\right) \text {. }
$$

Further, $L(\mathscr{J})=L(\mathscr{I})+\left|\boldsymbol{\beta}^{1}\right|-|\gamma|$ and

$$
\begin{aligned}
Q(\mathscr{J})=Q(\mathscr{I})+ & \sum_{k=2}^{\infty}\left[\left(\left|\beta_{2}^{1}\right|-\left|\gamma_{2}\right|\right)\left|\beta_{1}^{k}\right|+\left(\left|\left(\beta_{2}^{1}\right)^{-}\right|-\left|\left(\gamma_{2}\right)^{-}\right|\right)\left|\beta_{2}^{k}\right|+\right. \\
& \left.+\left(\left|\left(\beta_{2}^{1}\right)^{+}\right|-\left|\left(\gamma_{2}\right)^{+}\right|\right)\left|\left(\beta_{2}^{k}\right)^{-}\right|\right] .
\end{aligned}
$$

From (7.46) it follows

$$
\begin{gathered}
\left|\beta_{2}^{1}\right|-\left|\gamma_{2}\right| \leqq\left|u\left(b_{n+1}\right)-u\left(b_{n}\right)\right|, \\
\left|\left(\beta_{2}^{1}\right)^{ \pm}\right| \leqq\left|\left(\gamma_{2}\right)^{ \pm}\right|+\left|u\left(b_{n+1}\right)-u\left(b_{n}\right)\right| .
\end{gathered}
$$

Then

$$
\begin{aligned}
& L(\mathscr{J}) \leqq L(\mathscr{I})+\left|u\left(b_{n+1}\right)-u\left(b_{n}\right)\right|, \\
& Q(\mathscr{J}) \leqq Q(\mathscr{I})+\left|u\left(b_{n+1}\right)-u\left(b_{n}\right)\right| L(\mathscr{I}),
\end{aligned}
$$

and using (7.19), (7.20) we obtain

$$
\begin{gathered}
F_{1}(\mathscr{J}) \leqq F_{1}(\mathscr{I})+\left[1+14 K_{1} L(\mathscr{I})\right]\left|u\left(b_{n+1}\right)-u\left(b_{n}\right)\right| \leqq \\
\leqq F_{1}(\mathscr{I})+2\left|u\left(b_{n+1}\right)-u\left(b_{n}\right)\right| .
\end{gathered}
$$

Since $u\left(b_{n}\right)=u(n s), u\left(b_{n+1}\right)=u((n+2) s),(7.49)$ implies (7.45) for $i=1$.

If $b \in V(\mathscr{I}) \cap V(\mathscr{J})$ then $L(b ; \mathscr{J}) \leqq L(b ; \mathscr{I})+\left|\boldsymbol{\beta}^{1}\right|-|\gamma| \leqq L(b ; \mathscr{I})+\mid u\left(b_{n+1}\right)-$ $-u\left(b_{n}\right)$ and by means of (7.48), (7.19), (7.20) we get

$$
F_{2}(b ; \mathscr{J}) \leqq F_{2}(b ; \mathscr{I})+2\left|u\left(b_{n+1}\right)-u\left(b_{n}\right)\right| .
$$

Using (4.1) we see that $\left|U\left(b_{n+1}\right)-\tilde{U}\right| \leqq\left|U\left(a_{0, n+1}\right)-\tilde{U}\right|+\left(1+c^{*}\right)\left|\boldsymbol{\beta}^{1}\right|$ and since $L\left(b_{n+1} ; \mathscr{J}\right)=L\left(a_{0, n+1} ; \mathscr{I}\right)-|\gamma|$, the inequality

$$
\begin{aligned}
F_{2}\left(b_{n+1} ; \mathscr{J}\right) \leqq & c_{9}\left|U\left(a_{0, n+1}\right)-\tilde{U}\right|+c_{9}\left(1+c^{*}\right)\left|\boldsymbol{\beta}^{1}\right|-|\gamma|+ \\
& +L\left(a_{0, n+1} ; \mathscr{I}\right)+14 K_{1} Q(\mathscr{J})
\end{aligned}
$$


holds. Then (7.47), (7.48), (7.19) and (7.20) imply (7.50) for $b=b_{n+1}$ and hence (7.45) holds for $i=2$, too.

\section{ESTIMATES FOR THE APPROXIMATE SOLUTIONS}

Let us define

$$
\begin{gathered}
\bar{c}_{11} \equiv\left(2+c_{2}\right)\left(1+84 K_{1}\left(2+c_{2}\right) d_{8}\right), \quad \bar{c}_{12} \equiv c_{9}+2 \bar{c}_{11} ; \\
c_{14} \equiv \max \left[\frac{\bar{c}_{11}+2}{c_{1}}, \frac{\bar{c}_{12}+2}{c_{9}}\right] .
\end{gathered}
$$

The constants $d_{8}, c_{9}, \bar{c}_{1.1}, \bar{c}_{1.2}, c_{14}$ do not depend on $d_{50}$ but only on the function $F=(f, g)$ and the fixed vector $\tilde{U} \in \Omega_{F}$. Since $d_{50} \leqq d_{8}$ it is evident (compare (7.8), (7.17)) that

$$
c_{11} \leqq \bar{c}_{11}, \quad c_{12} \leqq \bar{c}_{12}
$$

Theorem 8.1. Let (5.1) hold with

$$
d_{50} \leqq \min \left[d_{8}, \frac{d_{8}}{4 c_{14}}, \frac{1}{56 K_{1}\left(2+\bar{c}_{11}\right)}\right] \equiv d_{51} .
$$

Then for each $a \in A, r>0$ the approximate solution $U_{r, a}$ is defined on the whole set $\bar{\Omega}$ and for $t \in\langle n s,(n+1) s), n \in \bar{N}, x \geqq 0$ it satisfies

$$
\begin{aligned}
& \left|U_{r, a}(x, t)-\tilde{U}\right| \leqq \\
& \leqq c_{14}\left(\left\|U_{0}-\tilde{U}\right\|+\left\|u_{1}-\tilde{u}\right\|+\underset{\langle 0, \infty)}{\operatorname{Var} U_{0}}+\underset{\langle 0,(n+1) s\rangle}{\operatorname{Var}} u_{1}\right) \leqq d_{8}, \\
& \operatorname{Var}_{\langle 0, \infty)} U_{r, a}(\cdot, t) \leqq \\
& \leqq c_{14}\left(\left\|U_{0}-\tilde{U}\right\|+\left\|u_{1}-\tilde{u}\right\|+\underset{\langle 0, \infty)}{\operatorname{Var} U_{0}}+\underset{\langle 0,(n+1) s\rangle}{\operatorname{Var}} u_{1}\right) \leqq d_{8} .
\end{aligned}
$$

Proof. For $\mathscr{J} \in[\mathscr{I}]$ starting at $b_{n}, n \in \overline{\mathrm{N}}$ we put $n(\mathscr{J}) \equiv n$. Further we fix $a \in A, r>0$ and denote $U=U_{r, a}$.

Let $[\mathscr{I}]^{0}$ be the set of all $\mathscr{I}$-curves $\mathscr{J} \in[\mathscr{I}]$ wit the properties

$$
\begin{gathered}
U \text { is defined along } \mathscr{J} ; \\
F_{1}(\mathscr{J}) \leqq \bar{c}_{11}\left(\left\|U_{0}-\tilde{U}\right\|+\left\|u_{1}-\tilde{u}\right\|+\right. \\
\left.+\underset{\langle 0, \infty)}{\operatorname{Var} U_{0}}+2 \sum_{i=1}^{n(\mathscr{F})}\left|u_{1, r}(i s)-u_{1, r}((i-1) s)\right|\right),
\end{gathered}
$$




$$
\begin{aligned}
& F_{2}(\mathscr{J}) \leqq \bar{c}_{1.2}\left(\left\|U_{0}-\tilde{U}\right\|+\left\|u_{1}-\tilde{u}\right\|+\right. \\
+ & \left.\underset{\langle 0, \infty)}{\operatorname{Var} U_{0}}+2 \sum_{i=1}^{n(\mathscr{J})}\left|u_{1, r}(i s)-u_{1, r}((i-1) s)\right|\right) .
\end{aligned}
$$

Now it is sufficient to show

$$
[\mathscr{I}]^{0}=[\mathscr{I}] \text {. }
$$

Indeed, each $(x, t) \in \bar{\Omega}$ lies in a strip $\Pi_{n}, n \in \overline{\mathrm{N}}$ and there exists an $\mathscr{I}$-curve $\mathscr{J} \subset \bar{\Pi}_{n}$. Since $\mathscr{J} \in[\mathscr{I}]^{0}$ by $(8.10),(8.7)$ implies that $U$ is defined on $\bar{\Pi}_{n}$, in particular at $(x, t)$. Further $n(\mathscr{J})=n$ and

$$
\sum_{i=1}^{n(\mathcal{F})}\left|u_{1, r}(i s)-u_{1, r}((i-1) s)\right| \leqq \underset{\langle 0,(n+1) s\rangle}{\operatorname{Var}} u_{1} \leqq d_{50} .
$$

Taking into account (8.11), (8.9) and (7.15) we see that $|U(b)-\tilde{U}| \leqq d_{8}$ for $b \in V(\mathscr{J})$. Therefore we can apply Lemma 7.6 to the point $b=(x, t)$ and with the help of (8.9), (8.11) we immediately obtain (8.5). Further Var $U(\cdot, t) \leqq F_{1}(\mathscr{J}) / c_{1}$ by Lemma 7.4 which yields $(8.6)$ by means of $(8.8),(8.11)$.

Thus it remains to prove $(8.10)$. Since the set $[\mathscr{I}]$ is partially ordered and satisfies the condition of minimality (see Lemma 6.3), it is sufficient to prove the following implication:

(8.12) If $\mathscr{J} \in[\mathscr{I}]$ and if $\mathscr{I} \in[\mathscr{I}]^{0}$ for each $\mathscr{I} \in[\mathscr{I}]$ such that $\mathscr{I}<\mathscr{J}$, then

$$
\mathscr{J} \in[\mathscr{I}]^{0} \text {. }
$$

Indeed, (8.12) implies (8.10) by the principle of Noether induction.

In particular $(8.12)$ requires $\mathcal{O} \in[\mathscr{I}]^{0}$. But this is an immediate consequence of Lemmas 7.4, 7.6 and (8.3). Further let $\mathscr{J} \in[\mathscr{I}], \mathscr{J} \neq \mathcal{O}$. Then there exists $\mathscr{I} \in[\mathscr{I}]$ such that $\mathscr{J}$ is a successor of $\mathscr{I}$. Consequently $\mathscr{I}<\mathscr{J}$ and therefore $\mathscr{I} \in[\mathscr{I}]^{0}$ by the induction assumption. Then again $|U(b)-\tilde{U}| \leqq d_{8}$ for $b \in V(\mathscr{I})$ and $F_{1}(\mathscr{I}) \leqq 4\left(\bar{c}_{11}+2\right) d_{50}$ by $(8.8),(8.11)$. (5.1). Hence if we put $c_{3}(\mathscr{I}) \equiv 4\left(\bar{c}_{11}+2\right)$, the assumptions of Lemmas 7.7-7.10 are satisfied and consequently (8.7) holds. Further, if $n(\mathscr{J})=n(\mathscr{I})$, then (8.8), (8.9) follow from (7.21), Lemma 7.7 or 7.8. For $n(\mathscr{J})=n(\mathscr{I})+1, n(\mathscr{I})$ even $(7.21)$ holds by Lemma 7.9 and since $u_{1, r}(n(\mathscr{J}) s)=$ $=u_{1, r}(n(\mathscr{I}) s)$ in this case, (7.21) again implies (8.8), (8.9). Finally if $n(\mathscr{J})=$ $=n(\mathscr{I})+1, n(\mathscr{I})$ odd, we deduce (8.8), (8.9) from (7.45), Lemma 7.10, and from the definition of $u_{1, r}$. Since obviously $0 \leqq n(\mathscr{J})-n(\mathscr{I}) \leqq 1$, the proof is complete.

Theorem 8.2. Let (5.1) hold with

$$
d_{50} \leqq \min \left[\frac{d_{51}}{8 c_{1.4}}, \frac{d_{51}}{4\left(1+c_{14}\right)}\right] \equiv d_{52}
$$

$\left(d_{51}\right.$ being introduced in (8.4)) and let

$$
c_{16} \equiv\left(1+17 c_{1.4}\right) c_{14} q_{0} .
$$


Then for all $t_{1} \geqq 0, t_{2} \geqq 0, r \geqq 0, a \in A$ it holds

$$
\begin{aligned}
& \int_{0}^{\infty}\left|U_{r, a}\left(x, t_{1}\right)-U_{r, a}\left(x, t_{2}\right)\right| \mathrm{d} x \leqq \\
& \leqq c_{16}\left(\left|t_{1}-t_{2}\right|+4 s\right)\left(\left\|U_{0}-\tilde{U}\right\|+\left\|u_{1}-\tilde{u}\right\|+\underset{\langle 0, \infty)}{\operatorname{Var} U_{0}}+\underset{\langle 0, \infty)}{\operatorname{Var}} u_{1}\right) .
\end{aligned}
$$

Proof. Fix $r_{0}>0, a^{0} \in A$ and put $U \equiv U_{r_{0}, a^{0}}$. By Theorem $8.1 U$ is defined on $\bar{\Omega}$ and satisfies (8.5), (8.6). We can suppose $t_{1}<t_{2}$ without loss of generality. Let $n_{0} \equiv \sup \left\{n \in \bar{N} ; n s \leqq t_{1}\right\}$ and let $q$ be the integer part of $\left(t_{2} / s\right)-n_{0}$ (so that $\left.s q \leqq t_{2}-t_{1}+s\right)$.

Now let us consider $m \in \overline{\mathrm{N}}$ such that $\left(m, n_{0}+q+1\right) \in Y$. Taking into account the construction of the approximate solutions, one can easily see that for each $x \in \Delta_{m}^{0} \equiv((m-1) r,(m+1) r\rangle \cap\langle 0, \infty)$ the values $U\left(x, t_{i}\right), i=1,2$, depend only on $U\left(\xi, n_{0} s\right)$ for $\xi \in \Delta_{m} \equiv\langle(m-q-2) r,(m+q+2) r\rangle \cap\langle 0, \infty)$ and further on $u_{1}(t), t \geqq n_{0} s$ in the case $m-q-2<0$. Let us define

$$
\begin{gathered}
\tilde{U}^{*} \equiv U\left(m r, n_{0} s\right) ; \\
U_{0}^{*}(\xi) \equiv\left\langle\begin{array}{ll}
U\left(\xi, n_{0} s\right) & \text { for } \xi \in \Delta_{m}, \\
\tilde{U}^{*} & \text { for } \xi \in\langle 0, \infty) \backslash \Delta_{m} ;
\end{array}\right. \\
u_{1}^{*}(\tau) \equiv\left\langle\begin{array}{lll}
u_{1}(\tau) & \text { for } \tau \geqq n_{0} s, & m-q-2<0, \\
u\left(m r, n_{0} s\right) & \text { for } \tau \geqq n_{0} s, & m-q-2 \geqq 0
\end{array}\right.
\end{gathered}
$$

and consider the approximate solutions $U_{r, a}^{*}$ of the problem

$$
\begin{gathered}
U_{l}+F(U)_{x}=0, \quad x \geqq 0, \quad t \geqq n_{0} s ; \\
U\left(x, n_{0} s\right)=U_{0}^{*}(x), \quad x>0 ; \quad u(0, t)=u_{1}^{*}(t), \quad t \geqq n_{0} s .
\end{gathered}
$$

(These solutions are constructed in the same way as before, only in the case $n_{0}$ odd we replace $Y$ by $Y^{*} \equiv\left\{(m, n) \in \mathrm{N}^{2} ; m+n\right.$ is odd $\}$.) Using (8.5), (8.6) we obtain

$$
\begin{aligned}
\left\|U_{0}^{*}-\tilde{U}\right\| & \leqq\left\|U\left(\cdot, n_{0} s\right)-\tilde{U}\right\| \leqq d_{51}, \\
\underset{\langle 0, \infty)}{\operatorname{Var}} U_{0}^{*} & =\underset{\Delta_{m}}{\operatorname{Var} U\left(\cdot, n_{0} s\right) \leqq d_{51},}
\end{aligned}
$$

and evidently $\operatorname{Var} u_{1}^{*} \leqq d_{51},\left\|u_{1}^{*}-\tilde{u}\right\| \leqq d_{51}$. Thus we can apply Theorem 8.1 to $U_{r, a}^{*}$ and hence $U_{r, a}^{*}$ are defined for all $x, t$; evidently

$$
U_{r_{0}, a^{0}}^{*}\left(x, t_{i}\right)=U\left(x, t_{i}\right), \quad x \in \Delta_{m}^{0}, \quad i=1,2 .
$$

Further, (8.5), (8.16), (8.13) yield

$$
\begin{gathered}
\left\|U_{0}^{*}-\tilde{U}^{*}\right\| \leqq 2\left\|U\left(\cdot, n_{0} s\right)-\tilde{U}\right\| \leqq 8 c_{14} d_{50} \leqq d_{51}, \\
\left\|u_{1}^{*}-\tilde{u}^{*}\right\| \leqq\left\|u_{1}^{*}-\tilde{u}\right\|+\left\|U\left(\cdot, n_{0} s\right)-\tilde{U}\right\| \leqq 4\left(1+c_{14}\right) d_{50} \leqq d_{51} .
\end{gathered}
$$


Consequently, we can replace $\tilde{U}$ by $\tilde{U}^{*}$ in Theorem 8.1 applied to $U_{r_{0}, a^{0}}^{*}$ and so (8.15), (8.17) imply

$$
\left|U\left(x, t_{1}\right)-U\left(x, t_{2}\right)\right| \leqq 2 c_{14}\left(\left\|U_{0}^{*}-\tilde{U}^{*}\right\|+\left\|u_{1}^{*}-\tilde{u}^{*}\right\|+\underset{\langle 0, \infty)}{\operatorname{Var}} U_{0}^{*}+\underset{\left\langle n_{0} s, \infty\right)}{\left.\operatorname{Var} u_{1}^{*}\right)} .\right.
$$

Let $d_{0} \equiv\left\|U_{0}-\tilde{U}\right\|+\left\|u_{1}-\tilde{u}\right\|+\operatorname{Var} U_{0}+\operatorname{Var} u_{1}$. Since

$$
\begin{aligned}
& \left\|U_{0}^{*}-\tilde{U}^{*}\right\| \leqq \underset{\Delta_{m}}{\operatorname{Var}} U\left(\cdot, n_{0} s\right) ; \underset{\langle 0, \infty)}{\operatorname{Var}} U_{0}^{*}=\underset{\Delta_{m}}{\operatorname{Var}} U\left(\cdot, n_{0} s\right) ; \\
& c_{15}(m) \equiv\left\|u_{1}^{*}-\tilde{u}^{*}\right\|+\underset{\left.<n_{0}, \infty\right)}{\operatorname{Var} u_{1}^{*}} \backslash \begin{array}{l}
=0 \text { if } m-q-2 \geqq 0, \\
\leqq\left(1+c_{14}\right) d_{0} \text { if } m-q-2<0
\end{array}
\end{aligned}
$$

and $\Delta_{m} \cap \Delta_{m^{*}}=\emptyset$ if $\left|m-m^{*}\right| \geqq 2(q+2)$, we have

$$
\begin{aligned}
& \sum_{m} c_{15}(m) \leqq(q+3)\left(1+c_{14}\right) d_{0}, \\
& \sum_{m} \underset{\Delta_{m}}{\operatorname{Var}} U\left(\cdot, n_{0} s\right) \leqq 2(q+3) \underset{\langle 0, \infty)}{\operatorname{Var} U\left(\cdot, n_{0} s\right),}
\end{aligned}
$$

and hence

$$
\begin{aligned}
\int_{0}^{\infty}\left|U\left(x, t_{2}\right)-U\left(x, t_{1}\right)\right| \mathrm{d} x & =\sum_{m} \int_{\Delta_{m^{0}}} \ldots \mathrm{d} x \leqq 8 r c_{14} \sum_{m}\left[\underset{\Delta_{m}}{\operatorname{Var}} U\left(\cdot, n_{0} s\right)+c_{15}(m)\right] \leqq \\
\leqq & (q+3)\left(1+17 c_{1.4}\right) c_{14} d_{0} .
\end{aligned}
$$

But $r(q+3) \leqq q_{0}\left(t_{2}-t_{1}+4 s\right)$ and thus (8.15) follows immediately from (8.18).

\section{CONVERGENCE OF THE APPROXIMATE SOLUTIONS}

From now on we shall suppose that $d_{50}$ satisfies (8.13), i.e. $d_{50} \leqq d_{52}$. Therefore Theorems 8.1, 8.2 hold, in particular $U_{r, a}$ are defined on $\bar{\Omega}$ for all $r>0, a \in A$. Let us denote

$$
\mathfrak{M} \equiv\left\{\varphi: \bar{\Omega} \rightarrow \mathrm{R} ; \varphi \in L_{\infty}(\Omega), \operatorname{supp} \varphi \text { is compact }\right\} .
$$

For $\varphi \in \mathfrak{M}, r>0, a \in A$ we define

$$
\begin{gathered}
\Delta(r, a, \varphi, n) \equiv \int_{0}^{\infty} \varphi(x, n s)\left[U_{r, a}(x, n s-0)-U_{r, a}(x, n s)\right] \mathrm{d} x, \quad n \in \mathrm{N} \\
\Delta(r, a, \varphi) \equiv \sum_{n=1}^{\infty} \Delta(r, a, \varphi, n)
\end{gathered}
$$

(the expressions in (9.1), (9.2) are vectors $\Delta=\left(\Delta_{1}, \Delta_{2}\right)$ ).

Lemma 9.1. Let $\varphi \in \mathfrak{M}, r>0, a \in A, n \in \mathrm{N}$. Then

$$
|\Delta(r, a, \varphi, n)| \leqq r\|\varphi\| 8 c_{14} d_{50} .
$$


Proof follows from the definition (5.13) of $U_{r, a}(\cdot, n s)$ and from Theorem 8.1 (see also [1]).

Let $\mathrm{P}=\prod_{(m, n) \in Y}\langle 0,1\rangle$ be the probabilistic space equipped with the product measure $\mathrm{d} p$ generated by the Lebesgue measure $\mathrm{d} x$ on $\langle 0,1\rangle$. For $r>0$ we define the mapping $Z_{r}: \mathrm{P} \rightarrow A(r)$ as follows: $Z_{r}\left(\left\{p_{m, n}\right\}\right)=\left\{a_{m, n}\right\}$ where $p_{m, n} \in\langle 0,1\rangle$ and $a_{m, n}=\left(\left(m-1+2 p_{m, n}\right) r, n s\right)$ for $m>0 ; a_{0, n}=\left(p_{m, n} r, n s\right)$. Further, we put

$$
\begin{aligned}
& \Delta_{0}(r, p, \varphi, n) \equiv \Delta\left(r, Z_{r}(p), \varphi, n\right), \\
& \Delta_{0}(r, p, \varphi) \equiv \Delta\left(r, Z_{r}(p), \varphi\right)=\sum_{n=1}^{\infty} \Delta_{0}(r, p, \varphi, n)
\end{aligned}
$$

and denote by $\mathfrak{M}(r), r>0$, the set of all functions $\varphi \in \mathfrak{M}$ such that for each $(m, n) \in$ $\in Y, \varphi$ is constant on the diamond

$$
\begin{gathered}
T_{m, n} \equiv\left\{(x, t) \in \bar{\Omega} ; x \in((m-1) r,(m+1) r\rangle,-\frac{s}{r}[x-(m-1) r]+n s \leqq t<\right. \\
<\frac{s}{r}[x-(m-1) r]+n s \text { for } x \leqq m r ; \frac{s}{r}[x-(m+1) r]+n s \leqq t< \\
\left.<-\frac{s}{r}[x-(m+1) r]+n s \text { for } x>m r\right\} .
\end{gathered}
$$

Lemma 9.2. Let $\varphi \in \mathfrak{M}(r), r>0, n_{1} \neq n_{2}, n_{i} \in \mathrm{N}, i=1$, 2. Then

$$
\int_{\mathrm{P}} \boldsymbol{\Delta}_{0}\left(r, p, \varphi, n_{1}\right) \Delta_{0}\left(r, p, \varphi, n_{2}\right) \mathrm{d} p=0 .
$$

Proof follows the same ideas as in the case of the Cauchy problem (see [1]) and so is omitted here.

For $\varphi \in \mathfrak{M}, r>0$ let us denote $n(r, \varphi) \equiv \inf \{n \in \mathrm{N} ; \varphi \equiv 0$ for $t>n s\}$. From now on we confine ourselves to $r$ of dyadic form, i.e. $r=2^{-k}, k \in \mathrm{N}$. Then $n(r, \varphi)=$ $=r^{-1} n(1, \varphi)$ and if $\varphi \in \mathfrak{M}(r)$ then $\varphi \in \mathfrak{M}\left(r^{*}\right)$ for all $r^{*}<r$. Further, Lemma 9.2 implies

$$
\left\|\Delta_{0}(r, \cdot, \varphi)\right\|_{L_{2}(\mathrm{P})}^{2}=\sum_{n=1}^{\infty}\left\|\Delta_{0}(r, \cdot, \varphi, n)\right\|_{L_{2}(\mathrm{P})}^{2}
$$

and using (9.3) we get the inequality

$$
\left\|\Delta_{0}(r, \cdot, \varphi)\right\|_{\boldsymbol{L}_{2}(\mathbf{P})}^{2} \leqq r n(1, \varphi)\left(\|\varphi\| 8 c_{14} d_{50}\right)^{2} .
$$

Consequently

$$
\lim _{r \rightarrow 0} \Delta_{0}(r, \cdot, \varphi)=0 \text { in }\left[L_{2}(\mathrm{P})\right]^{2} .
$$


Thus for each $\varphi \in \mathfrak{M}(r)$ we can choose a subsequence $\left\{r_{i}\right\}_{i=1}^{\infty}$ such that $\lim \Delta_{0}\left(r_{i}, p, \varphi\right)=0$ for a.a. $p \in \mathrm{P}$. Applying the diagonal process we can achieve this for a countable set of $\varphi$. Since there exists a sequence $\left\{\varphi_{i}\right\} \subset \bigcup \mathfrak{M}\left(2^{-k}\right)$ dense in $C_{0}^{2}$ in the $L_{\infty}(\bar{\Omega})$-norm, we see that the following lemma holds: $k \in \mathrm{N}$.

Lemma 9.3. There exist a sequence $\left\{k_{i}\right\}_{i=1}^{\infty} \subset \mathrm{N}$ and $N \subset \mathrm{P}$ such that mes $(N)=$ $=0, \lim _{i \rightarrow \infty} k_{i}=\infty$ and

$$
\lim _{i \rightarrow \infty} \Delta_{0}\left(2^{-k i}, p, \varphi\right)=0 \text { for all } p \in \mathrm{P} \backslash N, \quad \varphi \in C_{0}^{2} .
$$

Proof of Theorem 2.4. Let $d=d_{52}$ and let (2.12) hold. For fixed $p^{0} \in \mathrm{P} \backslash N$ we put

$$
U_{i} \equiv U_{r_{i}, Z_{r_{i}}\left(p^{0}\right)}, \quad i \in \mathrm{N}
$$

$\left(N, r_{i}=2^{-k_{i}}\right.$ are the set and the sequence from Lemma 9.3). By Theorem 8.1 we have

$$
\left\|U_{i}(\cdot, t)-\tilde{U}\right\| \leqq d_{8}, \underset{\langle 0, \infty)}{\operatorname{Var}} U_{i}(\cdot, t) \leqq d_{8}, \quad t \geqq 0, \quad i \in \mathrm{N} .
$$

Using Helly's theorem one can find a subsequence of $\left\{U_{i}(\cdot, t)\right\}, t \geqq 0$, converging in $\left[L_{1, l o c}\langle 0, \infty)\right]^{2}$. Applying the diagonal process we can achieve this for all rational $t>0$; let us denote this subsequence again by $\left\{U_{i}\right\}$. Now using Theorem 8.2 we can prove by a standard argument (see $[1])$ that $U_{i}(\cdot, t)$ converge in $\left.\left[L_{1, l o c}<0, \infty\right)\right]^{2}$ uniformly for bounded $t \geqq 0$; let the limit function be $U$. Then $\|U-\tilde{U}\| \leqq d_{8}$ by (9.8) and hence $U \in\left[L_{\infty}(\Omega)\right]^{2}, U(x, t) \in \Omega_{F}$ for a.a. $(x, t) \in \Omega$ and $F(U) \in\left[L_{\infty}(\Omega)\right]^{2}$. Further, it is seen that $F\left(U_{i}\right) \rightarrow F(U)$ in $\left[L_{1, l o c}(\Omega)\right]^{2}$. Therefore we obtain with the help of Lemma 5.1

$$
\begin{gathered}
\int_{\Omega}\left[\psi_{t} u+\psi_{x} f(U)\right] \mathrm{d} x \mathrm{~d} t+\int_{0}^{\infty} \psi(x, 0) u_{0}(x) \mathrm{d} x= \\
=\lim _{i \rightarrow \infty} \int_{\Omega}\left[\psi_{t} u_{i}+\psi_{x} f\left(U_{i}\right)\right] \mathrm{d} x \mathrm{~d} t+\int_{0}^{\infty} \psi(x, 0) u_{0}(x) \mathrm{d} x= \\
=\lim _{i \rightarrow \infty}\left\{\Delta_{1}\left(r_{i}, Z_{r_{i}}\left(p^{0}\right), \psi\right)+\int_{0}^{\infty} \psi(x, 0)\left[u_{0}(x)-u_{0, r_{i}}(x)\right] \mathrm{d} x\right\}, \quad \psi \in C_{00}^{2} ; \\
\int_{\Omega}\left[\varphi_{t} v+\varphi_{x} g(u)\right] \mathrm{d} x \mathrm{~d} t+\int_{0}^{\infty} \varphi(x, 0) v_{0}(x) \mathrm{d} x+\int_{0}^{\infty} \varphi(0, t) g\left(u_{1}(t)\right) \mathrm{d} t= \\
=\lim _{i \rightarrow \infty}\left\{\Delta_{2}\left(r_{i}, Z_{r_{i}}\left(p^{0}\right), \varphi\right)+\int_{0}^{\infty} \varphi(x, 0)\left[v_{0}(x)-v_{0, r_{i}}(x)\right] \mathrm{d} x+\right. \\
\left.+\int_{0}^{\infty} \varphi(0, t)\left[g\left(u_{1}(t)\right)-g\left(u_{1, r_{i}}(t)\right)\right] \mathrm{d} t\right\}, \varphi \in C_{0}^{2} .
\end{gathered}
$$


Now $\lim \Delta_{i}=0, i=1,2$ by Lemma 9.3 and since the remaining limits in (9.9), (9.10) are evidently zero, $U$ is a generalized solution of the mixed problem $(\mathscr{P})$. The inequalities (2.13)-(2.15) folow immediately from (8.5), (8.6), (8.15) (with $\left.c=\max \left(c_{14}, c_{16}\right)\right)$ and hence the proof is complete.

\section{References}

[1] J. Glimm: Solutions in the Large for Nonlinear Hyperbolic Systems of Equations, Comm. Pure Appl. Math. 18, 1965, 697-715.

[2] P. D. Lax: Hyperbolic Systems of Conservation Laws II, Comm. Pure Appl. Math. 10, 1957, 537-566.

[3] J. A. Smoller and J. L. Johnson: Global Solutions for an Extended Class of Hyperbolic Systems of Conservation Laws, Arch. Rational Mech. Anal. 32, 1969, 169-189.

[4] B. L. Rožděstvenskij and N. N. Janěnko: Systems of Quasilinear Equations (in Russian), Moskva 1968.

Author's address: 11382 Praha 1, Jungmannovo nám. 8, ČSSR (Výzkumný ústav mechanizace, automatizace a technologie výroby stavebních dílců). 